\title{
BFV-COMPLEX AND HIGHER HOMOTOPY STRUCTURES
}

\author{
FLORIAN SCHÄTZ
}

\begin{abstract}
We present an alternative approach to induced higher homotopy structures constructed by the 'basic perturbation lemma'. This approach is motivated by physical considerations and makes use of operads and their representations. As an application we prove that the BFV-complex controls the formal deformations of coisotropic submanifolds. This is established by identifying the $P_{\infty}$-algebra structure on the normal bundle of a coisotropic submanifold as a homotopy structure induced from the BFV-complex. Then we provide the connection to the geometric picture in the new framework.
\end{abstract}

\section{Contents}

1. Introduction

2. Transferring homotopy structures to cohomology

2.1. The BV formalism and effective actions

2.2. Perturbation theory for $L_{\infty}$-algebras

2.3. Differential complexes

2.4. Differential graded Lie algebras

2.5. Induced $L_{\infty}$-algebra structures on cohomology

2.6. Induced $A_{\infty}$-algebra structures on cohomology

3. Formal deformations of coisotropic submanifolds

The BF V-complex and Lie algebroids - a short review

3.2. Perturbation theory for the BFV-complex 20

3.3. The deformation problem and the BFV-complex 24

Appendix A. Proof of Lemma 2 29

Appendix B. Proof of Lemma 3

Appendix C. Proof of Proposition 1 31

Appendix D. Proof of Theorem 2 35

Appendix E. Globalisation of Theorem 4 36

References

Acknowledgement. We thank Alberto S. Cattaneo for many encouraging and inspiring discussions and his general support. We also thank D. Fiorenza, D. Indelicato, B. Keller, P. Mnëv, C. Rossi, S. Shadrin, J. Stasheff and M. Zambon for clarifying discussions and helpful remarks on a draft of this paper. Moreover

The author acknowledges partial support by the joint graduate school of mathematics of the ETH and the University of Zürich and by SNF-grant Nr.20-113439. This work has been partially supported by the European Union through the FP6 Marie Curie RTN ENIGMA (Contract number MRTN-CT-2004-5652) and by the European Science Foundation through the MISGAM program. 
we thank M. Bordemann, H.-C. Herbig, S.L. Lyakovich and A.A. Sharapov for explaining the globalisation of the BFV-complex to us.

\section{INTRODUCTION}

The deformation problem for coisotropic submanifolds was formulated by $\mathrm{Oh}$ and Park in the symplectic category in OP. They constructed an $L_{\infty}$-algebra structure on the normal bundle of a coisotropic submanifold. Given such an $L_{\infty^{-}}$ algebra one can define Maurer-Cartan elements and construct the corresponding deformation functor. The question arises whether this deformation problem is formal or more generally, if there is a 'resolution' of the deformation problem in terms of a differential graded Lie algebra which possesses a natural geometric or physical meaning. We give an affirmative answer to the second question in Theorem 4 (Subsection 3.2): it turns out that the BFV-complex, originally constructed to understand the quantization of gauge theories with open gauge algebras (see $\mathrm{BF}$ and $(\mathrm{BV}])$, controls the same deformation problem. In fact, we construct an $L_{\infty^{-}}$ algebra structure on the normal bundle of a coisotropic submanifold in terms of certain operads using only the data given by the BFV-complex. Next we prove that this $L_{\infty}$-algebra is quasi-isomorphic to the one on the BFV-complex. Then we demonstrate that the $L_{\infty}$-algebra given in terms of operads and the one constructed by $\mathrm{Oh}$ and Park coincide. Thus the equivalence between the deformation functors follows as a corollary.

We will first prove the equivalence of the two higher homotopy structures under the quite strong assumption that the normal bundle to the coisotropic submanifold under consideration is flat. In fact, we are in debt of M. Bordemann, H.-C. Herbig, S.L. Lyakovich and A.A. Sharapov who outlined the construction of an appropriate global version of the BFV-complex for arbitrary coisotropic submanifolds to the author who was not aware of this construction. This globalisation is outlined in $[\mathrm{LS}]$ and [B] and explained in more details in [H]. We will sketch the construction given in $[\mathrm{B}$ and explain how the local result extends in a straight forward manner to this globalisation of the BFV-complex. Moreover, we give a systematic treatment of the extented Poisson bracket in terms of homological perturbation theory.

Cattaneo and Felder used the $L_{\infty}$-algebra structure on the normal bundle of a coisotropic submanifold to study the deformation quantization of coisotropic submanifolds, see $\left[\mathrm{CF}\right.$. They recovered the $L_{\infty}$-algebra structure on the normal bundle as the tree-level part of the perturbative approach to the Poisson Sigma model. This approach comes along with a graphical description of the $L_{\infty}$-algebra structure that seems to have a tight relationship with the graphical description we present. We will make use of the presentation of the $L_{\infty}$-algebra structure on the normal bundle of a coisotropic submanifold given in $[\mathrm{CF}$. There it is shown that it can be obtained with help of an identification between certain graded manifolds that goes back to Roytenberg ( $[$ Roy $]$ ) and the so-called derived brackets as introduced by Th. Voronov $([\mathrm{V}])$.

On a more conceptual level, we try to present a connection between physics and higher homotopy structures. In fact, the results we described before can be seen as a particular example of a much more general connection between the differential homotopy structure on a complex and an induced homotopy structure on a 'nice' subcomplex (i.e. it must contain the whole cohomology). To understand and 
clarify this connection was one of the main motivations that led to this paper. As described above, the key observation in the proofs we give concerning the formal deformation problem for coisotropic submanifolds is that there is a totally algebraic way to obtain the higher homotopy structures on the normal bundle of a coisotropic submanifold. When one goes to the physical interpretation of these structures as an action that satisfies the classical master equation (on a graded vector space or a non-commutative analogue thereof), the procedure we use in calculating the induced homotopy structure is nothing but the construction of the effective action. If one tries to state this in a more mathematical framework, operads and their representations naturally arise. Moreover the procedure naturally delivers a morphism between the differential homotopy structure we start our construction with and the structure we induce on cohomology. Taking all this for granted it follows that the given and the induced structure are quasi-isomorphic exactly when the first-order part of the morphism that we obtain induces an isomorphism in cohomology. That this is indeed the case follows from the fact that we start with a quasi-isomorphism $\iota:(H, 0) \rightarrow(K,-D)$ of complexes which we perturb, so the result of this perturbation is still a quasi-isomorphism. We remark that this perturbation procedure is known as the 'basic perturbation lemma' of differential homological algebra - see GL for an introduction. More specifically, the physical and the homological approach produce the same structure in case of differential complexes. But in addition one obtains an interpretation of the new structure-maps in terms of certain operads and their representations on the (deformed) $\mathrm{Hom}_{h}$-operad. It is this very explicit connection between certain diagrams and the derived structure maps that allows us to identify the $L_{\infty}$-algebra structure we construct with the one constructed by Oh and Park.

We remark that the material presented in the Section 2 and in the Appendix is known to the experts. The explicit formulas for the structure maps of the induced homotopy structures in case of $A_{\infty}$-algebras are contained in [Me. These structure maps were interpreted as sums over trees by Kontsevich and Soibelman in [KS. These two papers also contain sketches of the proof that the new structure maps constitute a quasi-isomorphic $A_{\infty}$-algebra structure. Moreover, during a first draft of this paper was circulated, an improved version of [M] was brought to our attention which contains a discussion parallel to the one presented in Section 2.1 and 2.2 Moreover, claims equivalent to Theorem 2 in Subsection 2.3 are stated there and derived formally with help of path integrals.

\section{TRANSFERRING HOMOTOPY STRUCTURES TO COHOMOLOGY}

2.1. The BV formalism and effective actions. We will briefly review the BV formalism to deal with field theories, introduce the effective actions in this framework and give a short description of the Feynman rules that one needs to compute these effective actions in the perturbative approach. Throughout the whole article we will make use of the language of supergeometry (or more precisely the formalism developed to work with $\mathbb{Z}$-graded manifolds). The reader not familiar with this concept is adviced to consult [C2] and references therein for an introduction.

The BV formalism introduced by Batalin and Vilkovisky in [BV] is a systematic approach to the study of a huge class of field theories - we will basically follow C1] and [C2 in our review of their construction. Let $\mathcal{M}$ be a graded manifold 
which we should think of as the phase space of our theory. Consequently $T^{*}[-1] \mathcal{M}$ carries a canonical symplectic structure of degree -1 . We denote the corresponding Poisson bracket on $\mathcal{C}^{\infty}\left(T^{*}[-1] \mathcal{M}\right)$ by $(\cdot, \cdot)$. This bracket is the so called BV-bracket which has degree +1 . Moreover, we can define the following coboundary operator on $\mathcal{C}^{\infty}\left(T^{*}[-1] \mathcal{M}\right)$ after fixing a coordinate neighbourhood of $\mathcal{M}$ and considering the induced coordinates on a patch of $T^{*}[-1] \mathcal{M}$ :

$$
\Delta:=\frac{\partial^{2}}{\partial q^{i} \partial p_{i}}
$$

which is called the BV-differential. It is not canonically defined as a differential operator on the functions, however there is an geometric interpretation if one lets it operate on 'half-Berezinians' instead, see $\underline{\mathrm{K}}$ for details and $\underline{\mathrm{Se}}$ for a conceptual explanation. We will only deal with examples where $\mathcal{M}$ is a vector space and in this case one can identify functions and half-Berezinians without problems.

Later we will need a spectral parameter so that we can consider perturbations controlled by it. Consequently we allow all our functions to depend on some formal parameter $\epsilon$ too, although we will not indicate this dependence explicitly most of the time. The formal parameter $\epsilon$ corresponds to $-i \hbar$ in physical conventions.

A function $S \in \mathcal{C}^{\infty}\left(T^{*}[-1] \mathcal{M}\right)$ satisfies the quantum-master equation (QME) iff

$$
(S, S)+2 \epsilon \Delta(S)=0
$$

holds. The importance of the QME comes from the fact that it is equivalent to

$$
e^{\frac{S}{\epsilon}}
$$

being $\Delta$-closed and the following theorem due to A. Schwarz (see $[\mathrm{Sw}]$ or $[\mathrm{C} 2]$ for an explanation of the more general statement):

Theorem 1. Let $f$ be in $\mathcal{C}^{\infty}\left(T^{*}[-1] \mathcal{M}\right)$. Then

$$
\int_{\mathcal{L}} \Delta(f)=0
$$

for all Lagrangian submanifolds $\mathcal{L}$ of $T^{*}[-1] \mathcal{M}$, and

$$
\int_{\mathcal{L}} f
$$

is constant on cobordism classes of Lagrangian submanifolds iff $\Delta(f)=0$.

From the physical point of view the choice of a Lagrangian submanifold corresponds to gauge-fixing and the theorem asserts us that the integral (3) is gaugeinvariant iff $f$ is $\Delta$-closed.

We recall Losev's presentation $\underline{\mathrm{L}}$. From now on $S$ will always denote a function on $T^{*}[-1] \mathcal{M}$ of total degree 0 that satisfies the QME, which is called the action. Furthermore we assume that our graded manifold $\mathcal{M}$ is just a graded vector space which we denote by $K$. Now we might not be interested in the 'physics' on all of $K$ but rather on a particular subspace, which we denote by $H$. We choose a splitting

$$
K=H \oplus A
$$

of $K$. In particular, $K$ could be a differential complex, $H$ its cohomology embedded in $K$ and $A$ an acyclic subcomplex of $K$ (or more generally $H$ could be a subcomplex 
containing all the cohomology of $K$ ). We denote the image of $q \in K$ under this splitting by $q_{I R}+q_{U V} \in H \oplus A$, and apply the same notation for the induced splitting on $T^{*}[-1] K$. Physically speaking, we split our phase space in a part that contains the 'infrared' degrees of freedom and a part that contains the 'ultraviolet' degrees of freedom. When one is only interested in the physics on the infrared part, one should integrate out all the ultraviolet degrees of freedom. So we define

$$
e^{\frac{S_{e f f}}{\epsilon}}:=\int_{\mathcal{L}} e^{\frac{S}{\epsilon}}
$$

where $\mathcal{L}$ is a Lagrangian submanifold of $T^{*}[-1] A$.

Lemma 1. If $S$ satisfies the $Q M E$, so does $S_{\text {eff }}$ (with respect to $\Delta_{I R}$ ).

Proof. This is a consequence of the following computation:

$$
\Delta_{\mathrm{IR}} e^{\frac{S_{\text {eff }}}{\epsilon}}=\int_{\mathcal{L}} \Delta_{I R} e^{\frac{S}{\epsilon}}=\int_{\mathcal{L}} \Delta e^{\frac{S}{\epsilon}}=0
$$

where the equality between the two integrals follows from the fact that the integral of something $\Delta_{U V}$-exact over $\mathcal{L} \subset T^{*}[-1] A$ vanishes by Theorem 1 .

Doing field theory one is confronted with the problem to evaluate expressions like (4) over infinite-dimensional spaces. One way to surpass this problem is to work out formulas for these integrals in the finite-dimensional case and define (4) in the infinite dimensional case to be given by essentially the same formulas with the appropriate replacements of all the ingredients appearing in the formula. To do so one assumes that the expressions one wants to integrate are given by the expression for the Gaussian integral perturbed by some spectral parameter $\epsilon$. Then one works out formulas for all coefficients appearing in the expansion of the result in the formal parameter $\epsilon$. This leads to the so called Feynman rules for the perturbative approach to quantum field theory - we refer to $[\mathrm{BB}]$ and $[\mathrm{P}$ for more elaborate expositions. We will give a more precise version for the specific set of theories we are interested in later, but we give a rough explanation of the general rules to compute expression (4). We assume that the action is given by a polynomial in the coordinates on $T^{*}[-1] \mathcal{M}$ and that $S=B+\mathcal{O}(\epsilon)$ where $B$ is a non-degenerate quadratic form on $\mathcal{C}^{\infty}\left(T^{*}[-1] \mathcal{M}\right)$. The inverse of this form is called the propagator of the theory. In the part of the action $S$ that is at least of order $\epsilon$ there are terms that are cubic or of higher orders. Now one considers any connected (finite) graph containing vertices of valence equal to the order of the expressions occurring in $S-B=\mathcal{O}(\epsilon)$. To each such graph we associate the following function on $\mathcal{C}^{\infty}\left(T^{*}[-1] \mathcal{M}\right)$ : Every vertices of valence $k$ corresponds to the term in $S$ that is of polynomial order $k$. Along internal vertices one contracts with the inverse of $B$ and at the exterior vertices one places the inputs. This procedure results in a number, called the amplitude of the diagram. When we compute the sum of all these amplitudes over all possible diagrams we obtain $S_{\text {eff }}$.

2.2. Perturbation theory for $L_{\infty}$-algebras. Our next task is to specify the class of theories we are interested in. We will explain an interpretation of certain algebraic structure in terms of geometry and physics on graded manifolds. In fact, a huge part of the rest of this paper can be seen as an attempt to study the effective action (4) for physical theories that we obtain from this interpretation. It will turn out that the effective action which we will arrive at can be translated back in the 
algebraic language and as should be expected from the physical picture it has a very tight relation to the algebraic structure we started with. This Subsection is devoted to explaining in detail what we mean be all this in more mathematical terms.

We begin by describing the algebraic structure we are interested in, the so called $L_{\infty}$-algebras:

Definition 1. Let $V$ be a graded vector space together with a family of maps

$$
\left(m_{n}: S^{n}(V) \rightarrow V[1]\right)_{n \in \mathbb{N}} .
$$

Given such a family one defines the associated family of Jacobiators

$$
\left(J(n): S^{n}(V) \rightarrow V[2]\right)_{n \geq 1}
$$

by

$$
\begin{array}{r}
J(n)\left(x_{1} \cdots x_{n}\right):=\sum_{r+s=n} \sum_{\sigma \in(s, r)-\text { shuffles }} \operatorname{sign}(\sigma) m_{r+1}\left(m_{s}\left(x_{\sigma(1)} \otimes \cdots \otimes x_{\sigma(s)}\right) \otimes\right. \\
\left.\otimes x_{\sigma(s+1)} \otimes \cdots \otimes x_{\sigma(n)}\right)
\end{array}
$$

where $\operatorname{sign}(\cdot)$ is the Koszul sign, i.e. the one induced from the natural representation of $\Sigma_{n}$ on $S^{n}(V)$, and $(r, s)$-shuffles are permutations $\sigma$ of $(1, \cdots, r+s)$ such that $\sigma(1)<\cdots<\sigma(s)$ and $\sigma(s+1)<\cdots<\sigma(n)$. An $L_{\infty}$-algebra structure on $V$ is given by a family of maps as in (5) whose associated family of Jacobiators vanishes.

We allow 0 -ary operations $(\mathbb{R} \rightarrow V[1]) \cong V[1]$ and if this element vanishes one speaks of a flat $L_{\infty}$-algebra. Moreover, if $m_{n}=0$ for all $n>2$ we call the $L_{\infty^{-}}$ algebra an $L_{2}$-algebra. Giving this definition we basically follow Th. Voronov [V]. To connect this definition to the original one given by Stasheff [LSt] one has to apply the décalage-isomorphism

$$
\begin{aligned}
\operatorname{dec}_{n}: S^{n}(V) & \rightarrow \Lambda^{n}(V[-1])[n] \\
x_{1} \cdots x_{n} & \mapsto(-1)^{\sum_{i=1}^{n}(n-i)\left|x_{i}\right|} x_{1} \wedge \cdots \wedge x_{n}
\end{aligned}
$$

where $\left|x_{i}\right|$ is the degree of $x_{i}$ in $V$. Under this transition flat $L_{2}$-algebras correspond exactly to differential graded Lie algebras 1

Instead of giving the complicated - but explicit - condition in terms of Jacobiators one could also do the following: $S(V)$ carries a canonical structure of a cofree cocommutative coassociative coalgebra. Hence

$$
\operatorname{Hom}(S(V), V[k]) \cong \operatorname{Coder}(S(V))^{k}
$$

and the coderivations inherit a Lie-bracket from $\operatorname{End}(S(V))$. The condition on the Jacobiators is nothing but the condition that the corresponding coderivation commutes with itself with respect to this bracket. This observation is due to Stasheff.

\footnotetext{
${ }^{1}$ Our notion of $L_{\infty}$-algebra structure differs from the more traditional one where ' $L_{\infty}$-algebras' refer to what we would call 'flat $L_{\infty}$-algebras'. $L_{\infty}$-algebras with a non-vanishing 0 -ary operation were called 'curved $L_{\infty}$-algebras'. We prefer to think of $L_{\infty}$-algebras of some kind of space and as the notion of 'space' in differential geometry includes both flat and non-flat examples we allow the same to happen for $L_{\infty}$-algebras.
} 
To arrive at a more geometric picture we have to assume that $V$ is finite dimensional. Then

$$
\operatorname{Hom}(S(V), V[k]) \cong \operatorname{Hom}\left(V^{*}, S\left(V^{*}\right)[k]\right) \cong \operatorname{Der}\left(S\left(V^{*}\right)\right)^{k}
$$

and after suitable completion $S\left(V^{*}\right)$ can be identified with $\mathcal{C}^{\infty}(V)$. Hence we can interpret an $L_{\infty}$-algebra as a degree one vector field on $V$ and the condition on the Jacobiators translates into the condition that the corresponding vector field $X$ commutes with itself, i.e. $[X, X]=0$. Such a vector field is called cohomological.

Any vector field $X$ on $V$ can be interpreted as a function on $T^{*}[-1] V$ of degree $|X|-1$. So, starting with a cohomological vector field $X$ we obtain a function $S \in \mathcal{C}^{\infty}\left(T^{*}[-1] V\right)$ of degree zero. The Lie-bracket on the space of vector fields gets mapped to the BV-bracket. Consequently, $S$ satisfies the so-called classical master equation $(\mathrm{CME})$ :

$$
(S, S)=0
$$

which can be seen as the classical limit of the QME (1) for $\epsilon \rightarrow 0$ (or $\hbar \rightarrow 0$ ). If we assume that $S$ is additionally $\Delta$-closed - which is equivalent to requiring the cohomological vector field to be divergence-free - the QME is satisfied and we can apply the BV formalism as explained in Subsection 2.1.

So, what is the outcome of our perturbative defined effective action in the cases we are interested in? First we observe that the leading term of the action we start with must be quadratic as an element of $\mathcal{C}^{\infty}\left(T^{*}[-1] \mathcal{M}\right)$. Since it comes from a cohomological vector field and hence is linear in the coordinates along the fibres of $T^{*} K$, the leading part of $S$ in the expansion with respect to $\epsilon$ must also be linear in the coordinates on $K$. When we go back to the definition of $L_{\infty}$-algebras we gave in Definition 1 we see that this linearity condition amounts to require that the leading term must be a map $K \rightarrow K[1]$. From $S=d+\mathcal{O}(\epsilon)$ it follows that $d \circ d=0$, so $d$ must be a coboundary operator on $K$. Next we have to find a convenient gauge and a propagator for this differential. It turns out that there is a natural choice L: first fix an embedding of the homology of $(K, d)$ into $K$ :

$$
\iota: H \hookrightarrow K
$$

and choose a complement of $\iota(H)$ in $K$ that is an acyclic subspace. So one obtains the splitting $K=H \oplus A$ mentioned before. Next fix a homotopy for $d$, i.e. a linear map $h: K \rightarrow K[-1]$ such that

$$
d \circ h+h \circ d=i d-\Pi_{H}
$$

where $\Pi_{H}$ is the projection of $K$ to $H$ along $A$. The choice of gauge is

$$
\mathcal{L}:=N^{*}[-1](A \cap \operatorname{ker}(h)),
$$

the conormal bundle of the kernel of the homotopy in $A$. Clearly $\mathcal{L}$ is Lagrangian and when one computes the restriction of $S$ to $N^{*}[-1](A \cap \operatorname{ker}(h))$ it turns out that

$$
h \circ d=i d_{A}
$$

so one can use $h$ as the propagator of our theory. Diagrammatically, $h$ goes between a $p$ and a $q$ and we choose the direction of the arrow to go from $p$ to $q$ : 


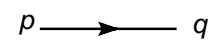

Moreover, all parts of $S$ are linear in the fibre-coordinates, so for each $m_{n}$ : $S^{n}(V) \rightarrow V[1]$ we obtain a vertex of valence $n+1$ with $n$ incoming arrows and 1 outgoing (the orientation is chosen such that it is consistent with the one for the homotopy):

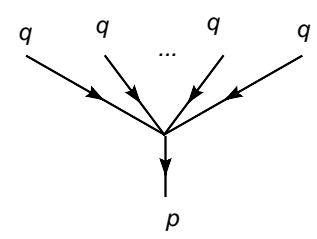

Suppose that one of the diagrams has a 'p' at an external vertex. Then it must be placed at the output of one of the n-ary operations pictured above (as we do not allow propagators attached to external vertices). If one of the 'inputs' of this n-ary operation is connected to another 'p' by a propagator this 'p' cannot be an external vertex (again because we do not allow propagators at external vertices). From this and the connectedness-condition it follows immediately that the 'p' we started with must be the only 'p' at an external vertex in the diagram. All these diagrams are called tree-level diagrams for obvious reasons and the give a function on $T^{*}[-1] H$ that is linear in the fibre coordinates and which we denote by $S_{\text {tree }}$. The only other connected diagrams that are possible are tree-diagrams where one joins the 'output' with one of its 'inputs'. This gives a function on $T^{*}[-1] H$ that does not depend on the fibre coordinates at all and which we denote by $S_{\text {loop }}$. What does $S_{\text {eff }}=S_{\text {tree }}+S_{\text {loop }}$ imply for the QME which we know is satisfied for $S_{\text {eff }}$ by Lemma 1]. When one orders the QME for $S$ by polynomial degrees in 'p' one obtains two separate equations:

$$
\begin{aligned}
\left(S_{\text {tree }}, S_{\text {tree }}\right) & =0 \\
\left(S_{\text {tree }}, S_{\text {loop }}\right)+\epsilon \Delta S_{\text {tree }} & =0 .
\end{aligned}
$$

This separation of the QME is also contained in [M]. The first equation tells us that we obtain an action on tree-level that satisfied the CME. It is obvious that $S_{\text {eff }}$ and hence $S_{\text {tree }}$ and $S_{\text {loop }}$ have the same total degree as $S$. Moreover $S_{\text {tree }}$ is linear in the coordinates along the fibres, so it can be interpreted as a cohomological vector field on $H$ and thus gives an $L_{\infty}$-algebra structure there. The second equation roughly relates the composition of

$$
\begin{aligned}
S_{\text {tree }} \in \mathcal{C}^{\infty}(H) \otimes H^{*} \cong & S\left(H^{*}\right) \otimes H \cong \operatorname{Hom}(S(H), H) \cong \operatorname{Coder}(S(H)) \text { and } \\
& S_{\text {loop }} \in S\left(H^{*}\right) \cong \operatorname{Hom}(S(H), \mathbb{R}) \\
\text { with }\left(x_{1} \otimes \cdots \otimes x_{n}\right) \mapsto & \operatorname{tr}\left(S_{\text {tree }}\left(x_{1} \otimes \cdots \otimes x_{n} \otimes(\cdot)\right)\right) .
\end{aligned}
$$

From the mathematical viewpoint some caution is in place because what we have done so far assumes the existence of a Lebesgue-like measure over infinitedimensional spaces. However the calculations are strong indications that one can extract something mathematical rigorous out of this procedure. To do this for at least an interesting class of examples is the content of the next four subsections. Basically, we will hide the physical interpretation of $L_{\infty}$-algebra structures and the use of perturbation theory to construct a new one in our treatment. Instead we 
will start from a flat $L_{2}$-algebra structure, construct an operad and a representation out of it and prove that we obtain an $L_{\infty}$-algebra structure together with an $L_{\infty^{-}}$ algebra morphism (we will give an explicit definition of these morphisms adapted to the examples we will investigate later).

2.3. Differential complexes. We start with the simplest example that fits into our scheme of theories described in Subsection 2.2 - the case of a differential

$$
\begin{aligned}
& D_{\epsilon}: \quad K \rightarrow K[1] \\
& D_{\epsilon}=\delta_{0}+\epsilon \delta_{1}+\epsilon^{2} \delta_{2}+\cdots
\end{aligned}
$$

where $\delta_{i}: K \rightarrow K[1]$ for all $i \geq 0$ (to be totally precise we should write $D_{\epsilon}$ : $K[[\epsilon]] \rightarrow K[[\epsilon]][1]$ but as usually we suppress the $\epsilon$-dependence where it does not harm). It is convenient to write $D_{R}:=\epsilon \delta_{1}+\epsilon^{2} \delta_{2}+\cdots$ which can be though of as the perturbation of the differential $\delta_{0}: K \rightarrow K[1]$.

Next we fix a homotopy $h$ for $\delta_{0}$ and from this we obtain also a projection to $H_{\delta_{0}}=: H$ by formula (7). The rules for the computation of the effective action have to be modified a little bit as we also allow 'bivalent' vertices what is not usually the case, but it can be done (basically, we perturb the propagator here - if $\delta_{0}$ can be inverted so can $\delta_{0}+\epsilon(\cdots)$ and one computes this inverse with the help of a geometric series).

From the mathematical side it suffices to define the following procedure and prove that it works: First we define the 'decorated line operad' $\mathbb{L}_{d}$. It is simply given by all oriented lines that are decorated by a natural number $>0$. That these form an operad is trivial (the reader not familiar with operads is adviced to skip through the first Chapter of [MSS] - we simply define the join of a line that is decorated by $m$ and a line that is decorated by $n$ as the line that is decorated by $m+n$. We denote this very simple operad by $\mathbb{L}_{d}$. Next we define a new operadic structure on $\operatorname{Hom}(K, K)$ by defining that 'composition' of two maps $\phi_{1}$ and $\phi_{2}$ should not be given by $\phi_{2} \circ \phi_{1}$ but by $\phi_{2} \circ h \circ \phi_{1}$ instead. This definition naturally extends to a new operadic structure on $\operatorname{Hom}(T(K), K)$ which we denote by $\operatorname{Hom}_{h}(T(K), K)(T(K)$ denotes the tensor algebra of $K)$. The computation of amplitudes from Feynman rules can be encoded in a representation of $\mathbb{L}_{d}$ on $\operatorname{Hom}_{h}(K, K)$ by defining

$$
\begin{aligned}
\Phi: \mathbb{L}_{d} & \rightarrow \operatorname{Hom}_{h}(K, K) \\
\text { line decorated with } n & \mapsto(-1)^{n} D_{R} \circ\left(h \circ D_{R}\right)^{n-1}
\end{aligned}
$$

Obviously, this defines a representation of operads (up to the fact that we have slightly modified the Hom-operad). Observe that $\Phi$ (line decorated with $n): H \rightarrow$ $H$ is a map of degree 1 again. Next we define the 'embedding'

$$
\begin{aligned}
C: \operatorname{Hom}_{h}(K, K) & \rightarrow \operatorname{Hom}(H, H) \\
\psi & \mapsto \Pi_{H} \circ \psi \circ \iota
\end{aligned}
$$

where $\Pi_{H}: K \rightarrow H$ is the projection of $K$ to the cohomology $H$ of $\delta_{0}$ obtained from formula (7) and $\iota: H \rightarrow K$ is the corresponding embedding.

Lemma 2. The 'quantum differential' $\mathcal{D}_{\epsilon}$ defined as the embedding of

$$
\begin{aligned}
\widetilde{\mathcal{D}_{\epsilon}: K[[\epsilon]]} & \rightarrow K[[\epsilon]][1] \\
\widetilde{\mathcal{D}_{\epsilon}} & :=\sum_{L \in \mathbb{L}_{d}} \Phi(L)
\end{aligned}
$$


is a coboundary operator.

Moreover the chain map

$$
\left(i d+h \circ \widetilde{\mathcal{D}_{\epsilon}}\right) \circ \iota:\left(H, \mathcal{D}_{\epsilon}\right) \rightarrow\left(K,-D_{\epsilon}\right)
$$

is a quasi-isomorphism of complexes.

We remark that $\widetilde{\mathcal{D}_{\epsilon}}$ is well-defined as a formal series in $\epsilon$ as $D_{R}$ is of order $\geq 1$ in $\epsilon$ and so $\Phi(L)$ is of order $\geq n$ in $\epsilon$ for $L$ decorated by $n$. A careful proof of Lemma 2 with help of some kind of extended graphical calculus is contained in the Appendix.

There are two specific cases we will investigate in more detail now. The first one is the situation in which $D_{\epsilon}=\delta_{0}+\epsilon \delta_{1}$. We will show that the quantum coboundary operator $\mathcal{D}_{\epsilon}$ can be used to deduce a spectral sequence for this $D_{\epsilon}$ as a homotopyinvariant. The spectral sequence works as follows: Given a differential $D_{\epsilon}=\delta_{0}+\epsilon \delta_{1}$ we can consider the following short exact sequence of complexes

$$
0 \longrightarrow\left(K[[\epsilon]], D_{\epsilon}\right) \stackrel{\epsilon}{\longrightarrow}\left(K[[\epsilon]], D_{\epsilon}\right) \stackrel{\bmod (\epsilon)}{\longrightarrow}\left(K, \delta_{0}\right) \longrightarrow 0
$$

which leads to a long exact sequence in cohomology that can be interpreted as an exact couple (see $[\mathrm{BT}]$ for details) which in turn leads to the spectral sequence.

On the other hand we can construct the quantum coboundary operator $\mathcal{D}_{\epsilon}$ which however heavily depends on the choice of the homotopy $h$. But we can try to construct something out of it that does not depend on the homotopy. The quantum coboundary operator has an expansion in $\epsilon$ that is given by

$$
\mathcal{D}_{\epsilon}=-\epsilon\left(\Pi_{H} \circ \delta_{1}\right)+\epsilon^{2}\left(\Pi_{h} \circ \delta_{1} \circ h \circ \delta_{1}\right)-\cdots
$$

The leading term does not depend on the homotopy and by power counting it follows that it must be a coboundary operator itself. So we can take the cohomology of $\left(H[[\epsilon]], \Pi_{H} \circ \delta_{1}\right)$. The second term in the expansion factors through to this cohomology and is a coboundary operator there, so can take the cohomology with respect to it. In general, the $n$-th part of the expansion of $\mathcal{D}_{\epsilon}$ in powers of $\epsilon$ is a coboundary operator up to the terms of order $<n$. So we can successively take the cohomologies. We claim that by doing so we reproduce exactly the sheets of the spectral sequence we introduced above. In particular this means that the procedure of iteratively taking cohomologies does not depend on the fixed homotopy $h$ and that we obtain the spectral sequence as a homotopy invariant of the quantum complex $\left(H[[\epsilon]], \mathcal{D}_{\epsilon}\right)$ (again, the sign issue arises: to really obtain the same signs as in the spectral sequence we should have started from $-D_{\epsilon}$ ).

Lemma 3. The spectral sequence associated to $\left(K[[\epsilon]], D_{\epsilon}\right)$ is a homotopy invariant of $\left(H[[\epsilon]], \mathcal{D}_{\epsilon}\right)$.

The fact that some spectral sequences can be obtained as homotopy invariants with the help of the effective action was mentioned in $\mathrm{L}$. Again, a detailed proof can be found in the Appendix.

The second situation that is of special interest for us is when there is a bigrading of $K=\bigoplus_{p, q} K^{q, p}$ that satisfies some special properties. We call the first degree the ghost-degree and the second one the ghost-momentum degree. $K$ should be bounded from below and above with respect to both degrees and the ghost-degree is $\geq 0$ while the ghost-momentum degree $\leq 0$. The maps $\delta_{i}: K \rightarrow K[1]$ should 
have ghost degree $i$ and ghost-momentum degree $1-i$, in particular $\delta_{0}$ is of ghost degree 0 and of ghost-momentum degree +1 . Consequently the homotopy $h$ has ghost-momentum degree -1 . We assume that the cohomology of $K$ with respect to $\delta_{0}$ is concentrated in the part of $K$ that has ghost-momentum degree 0 . By a simple counting of ghost-momentum degrees this implies that the quantum differential $\mathcal{D}_{\epsilon}$ only consists of $-\Pi_{H} \circ \delta_{1}$. Moreover, we can set $\epsilon=1$ since we only deal with a finite number of maps due to the boundedness-conditions on $K$. So Lemma 2 immediately implies

Corollary 1. For a complex $K$ as described above, the chain map

$$
\left(i d+h \circ \widetilde{\mathcal{D}}_{\epsilon=1}\right) \circ \iota:\left(H_{\delta_{1}},\left[\delta_{2}\right]\right) \rightarrow\left(K,-D_{\epsilon=1}\right)
$$

is a quasi-isomorphism.

Hence we recover the well-known fact that when the first sheet of the spectral sequence collapses, the next sheet is isomorphic to the cohomology of the total differential.

All the above material concerns the tree-part of the perturbative calculation of the induced $L_{\infty}$-algebra structure. But one could also investigate the loopstructure. However, in the case of differential complexes, this does not contain any information. The differentials all have degree 1 , so their traces vanish and the loop-diagrams give only numbers, so equation (9) reduces to something vacuous.

We remark that the results of this Subsection are all equivalent to the 'basic perturbation lemma' of differential homological algebra, see GL. In fact we just recovered the formulas for the perturbed differential and the perturbed quasiisomorphism that is given by this lemma. Moreover, in principle, the tools presented so far already suffice to deal with deformations of $L_{\infty}$ - or $A_{\infty}$-algebra structures: we interpret these structures as a coderivation on $S(K)$ or $T(K)$ which are boundary operators again. Then we perturb this differential and apply the results of this Subsection to obtain perturbed differentials on $S(H)$ or $T(H)$ respectively. But these can be reinterpreted as an $L_{\infty}$-algebra or $A_{\infty}$-algebra structure, see GL for more details.

We will demonstrate that one can extend the interpretation of the new structure maps in terms of operads and their representations on deformed $\mathrm{Hom}_{h}$-operads in the next two Subsections. This very constructive interpretation will turn out crucial when we want to compare the construction we give to the one of Oh and Park. Moreover we will extend this diagrammatic approach to the setting of differential graded associative algebras.

2.4. Differential graded Lie algebras. The next example concerns differential graded Lie algebras which is equivalent to consider flat $L_{2}$-algebras in the conventions we use. We denote the two structure maps by $d: K \rightarrow K[1]$ and $[\cdot, \cdot]: S^{2}(K) \rightarrow K[1]$ and we are interested in the perturbation given by $d+\epsilon[\cdot, \cdot]=$ : $d+[\cdot, \cdot]_{\epsilon}$. As before, fix a homotopy $h$ for $d$ and denote the corresponding projection to the cohomology of $d$ by $\Pi_{H}: K \rightarrow H$. The perturbation part of the differential graded Lie algebra contains a structure that is cubic as a polynomial function on $T^{*}[-1] K$ so our diagrammatic expansion contains trivalent vertices. More precisely 
we give the following construction: let $\mathbb{T}$ be the operad of oriented rooted trees, i.e. finite connected graphs without loops of any kind that only consist of oriented edges and trivalent interior vertices with two incoming edges and one outcoming one. Consequently, there are two kind of exterior vertices: ones with an outgoing edge - we call these leaves - and exactly one with an incoming edge that we call the root:

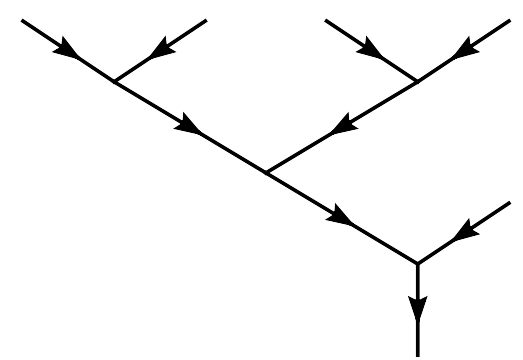

We only allow diagrams with at least two leaves. Clearly we have a decomposition

$$
\mathbb{T}=\bigsqcup_{n \geq 2} \mathbb{T}(n)
$$

where $\mathbb{T}(n)$ denotes the set of trees with exactly $n$ leaves. Observe that the orientation is given by an association of two numbers to any pair of edges with the same vertex as their target that tells us which of the two edges is the 'right' and which is the 'left' one. We will denote the set of unoriented trees by $[\mathbb{T}]$. There is a natural projection

$$
[\cdot]: \mathbb{T} \rightarrow[\mathbb{T}]
$$

that respects the decomposition of $\mathbb{T}$ and that of $[\mathbb{T}]$ :

$$
[\mathbb{T}]=\bigsqcup_{n \geq 2}[\mathbb{T}](n)=\bigsqcup_{n \geq 2}[\mathbb{T}(n)] .
$$

Moreover $\mathbb{T}$ clearly is an operad with respect to the joining of the root of one tree with a leaf of another tree. Next we construct a representation of this operad on the deformed homomorphism-operad $\operatorname{Hom}_{h}(T(K), K[1])$, where we replace ordinary composition by oho. Let

$$
\Phi: \mathbb{T} \rightarrow \operatorname{Hom}_{h}(T(K), K[1])
$$

be defined by the following rule: associate $[\cdot, \cdot]_{\epsilon}$ to every internal trivalent vertex and $h$ to every internal edge (an edge is called internal if it is not connected to a leave or to the root). Place the input $x_{1} \otimes \cdots \otimes x_{n}$ at the leaves which obtain a natural ordering thanks to the orientation of all the internal vertices. The output will by an element of $K$. Clearly this map has degree 1 and it defines a representation. We have to symmetrize the maps as we want to arrive at an $L_{\infty}$-algebra structure in the end. So we define

$$
\begin{aligned}
\Omega: \mathbb{T}(n) & \rightarrow \operatorname{Hom}_{h}\left(T^{n}(K), K[1]\right) \\
(\text { tree }) & \mapsto \frac{1}{|\operatorname{Aut}([\operatorname{tree}])|} \sum_{\sigma \in \Sigma_{n}} \operatorname{sign}(\sigma) \sigma^{*} \Phi(\text { tree })
\end{aligned}
$$

where Aut $(\cdot)$ denotes the automorphism group of an unoriented tree and $\sigma^{*} \Phi($ tree) denotes the pull-back of the map $\Phi($ tree $): T^{n}(K) \rightarrow K[1]$ by the bijection of $T^{n}(K)$ 
induced by $\sigma$. Observe that $\Omega$ is graded symmetric and moreover it is invariant under a change of the orientation of the tree. So it descends to a map

$$
[\mathbb{T}] \rightarrow \operatorname{Hom}_{h}(S(K), K[1])
$$

which we also denote by $\Omega$. We define

$$
\begin{aligned}
& \widetilde{M}_{n}: \quad S^{n}(K) \rightarrow K[1] \\
& \widetilde{M}_{n}:=\sum_{[G] \in[\mathbb{T}(n)]} \Omega([G])
\end{aligned}
$$

and we denote the embedding of $\widetilde{M}_{n}$ by $M_{n}: S^{n}(H) \rightarrow H[1]$ and claim

Proposition 1. The family of maps $\left(M_{n}: S^{n}(H) \rightarrow H[1]\right)_{n \geq 1}$ defines an $L_{\infty}$ algebra structure on $H$. Moreover this $L_{\infty}$-algebra structure is quasi-isomorphic to the one on $K$ we started with (with the usual sign-shift from $d$ to $-d$ ).

A proof using a version of the graphical calculus is contained in the Appendix.

2.5. Induced $L_{\infty}$-algebra structures on cohomology. We will discuss the diagrammatical and combinatorial arguments that we will need to construct the $P_{\infty^{-}}$ algebra structure on the normal bundle of a coisotropic submanifold in the next Section. We remark that the following works: Start with a differential complex $(K, d)$ and some quasi-isomorphic subcomplex $(H, \delta)$ together with a fixed quasiisomorphism. Then perturb the $L_{\infty}$-algebra structure $d$ to a new $L_{\infty}$-algebra structure $d+\mathcal{O}(\epsilon)$. One can compute the corresponding perturbation of the $L_{\infty}$-algebra -structure $\delta$ on $H$ and the perturbation of the original $L_{\infty}$-quasi-isomorphism between $H$ and $K$ with help of the methods outline in the last Section. All this is canonical up to the choice of a homotopy $h$ which also amounts to a choice of a specific quasi-isomorphism between $K$ and $H$.

This approach is equivalent to the approach presented in GL. The additional information is the statement that the structure maps of the perturbed structure on $H$ have a natural interpretation in terms of operads and their representations on the deformed $\mathrm{Hom}_{h}$-operad.

We present a proof of this statement only under the assumption that the deformed $L_{\infty}$-algebra structure on $K$ is a flat $L_{2}$-algebra structure and that the quasiisomorphic subcomplex is the cohomology of $K$ suitably embedded in $K$.

Proposition 2. Let $\left(K, \delta_{0}\right)$ be a complex with cohomology $H$. Fix a homotopy $h$ of $\delta_{0}$, i.e. a linear map $h$ that satisfies

$$
\delta_{0} \circ h+h \circ \delta_{0}=i d-\Pi_{H} .
$$

Using $\Pi_{H}$ one constructs a quasi-isomorphism $\iota: H \rightarrow K$. Let $\delta_{0}+\mathcal{O}(\epsilon)$ be any flat $L_{2}$-algebra structure on $K[[\epsilon]]$.

Then there is a canonical deformation of the trivial $L_{\infty}$-algebra structure on $H[[\epsilon]]$ and a deformation of the quasi-isomorphism $\iota$ that is a quasi-isomorphism between the perturbed $L_{\infty}$-algebra structure on $K[[\epsilon]]$ and the perturbed one on $H[[\epsilon]]$.

We introduced the operad of decorated trees $\mathbb{T}_{d}$ which contains all trees but all the edges (internal and external ones) are decorated by some natural number $\geq 0$. We exclude the graph that consist of a single line that is decorated by 0 . Clearly, this is a straightforward generalization of the line operad $\mathbb{L}$ and the tree operad $\mathbb{T}$ 
we used in the previous Subsection. $K[[\epsilon]]$ is a flat $L_{2}$-algebra that is a perturbation of the coboundary operator $\delta_{0}$. We denote the total perturbed differential by $D_{\epsilon}$ and the perturbation part by $D_{R}$. The bracket is denoted by $[\cdot, \cdot]_{\epsilon}$. As before we construct a representation

$$
\Phi: \mathbb{T}_{d} \rightarrow \operatorname{Hom}_{h}(T(K), K[1])
$$

from these data: put a $[\cdot, \cdot]_{\epsilon}$ at every trivalent vertex and $n-D_{R}$ 's on edges decorated by $n$. Between all these operations a homotopy $h$ is placed. Then similar to Subsection 2.4 we define

$$
\Omega:\left[\mathbb{T}_{d}\right] \rightarrow \operatorname{Hom}_{h}(S(K), K[1])
$$

by symmetrizing $\Phi$ and dividing by the number of elements in the automorphism group of the decorated unoriented tree (two decorated trees are equivalent if the are equal up to orientation and if the decorations are preserved). Again

$$
[\cdot]: \mathbb{T}_{d} \rightarrow\left[\mathbb{T}_{d}\right]
$$

is the projection from an oriented decorated tree to the corresponding unoriented one. Given $\Omega$ it is straight forward to define

$$
\begin{aligned}
& \widetilde{M_{n}}: \quad S^{n}(K) \rightarrow K[1] \\
& \widetilde{M_{n}}:=\sum_{[G] \in\left[\mathbb{T}_{d}(n)\right]} \Omega([G]) .
\end{aligned}
$$

Denote the embedding of these maps by $M_{n}$. As a refinement of Proposition 2 we claim

Theorem 2. The family of maps $\left(M_{n}: S(H) \rightarrow H[1]\right)_{n \geq 1}$ defines an $L_{\infty}$-algebra structure on $H$ that is quasi-isomorphic to the one on $\left(\bar{K},-D_{\epsilon},[\cdot, \cdot]_{\epsilon}\right)$. The quasiisomorphism is given explicitly by the family of maps $\left(\iota+h \circ \widetilde{M}_{1}, h \circ \widetilde{M}_{2}, h \circ \widetilde{M}_{3}, \cdots\right)$.

A totally constructive proof of this statement is given in the Appendix.

2.6. Induced $A_{\infty}$-algebra structures on cohomology. We give a treatment of $A_{\infty}$-algebra structures in our setting. From a physical point of view this should correspond to the computation of the tree-level of an effective action of a quantum field theory on a non-commutative space. This follows from the fact that an $A_{\infty^{-}}$ algebra structures can be interpreted as a coderivation on the tensor algebra that is a coboundary operator (this observation is due to Stasheff). However, there is no direct interpretation of a tensor algebra in terms of functions on some (gradedcommutative) space. Nevertheless, we will see that one can formally carry out analogous constructions to the one presented in the previous Subsection and obtain parallel results. First we give the relevant definitions:

Definition 2. Let $V$ be a graded vector space together with a family of maps

$$
\left(z_{n}: T^{n}(V) \rightarrow V[1]\right)_{n \in \mathbb{N}} .
$$

Given such a family one defines its family of associators

$$
\left(A(n): T^{n}(V) \rightarrow V[2]\right)_{n \geq 1}
$$


by

$$
\begin{array}{r}
A(n)\left(x_{1} \cdots x_{n}\right):=\sum_{r+s=n} \sum_{i=0}^{n-s+1}(-1)^{\left|x_{1}\right|+\cdots+\left|x_{i-1}\right|} z_{r+1}\left(x_{1} \otimes \cdots \otimes x_{i-1} \otimes\right. \\
\left.\otimes z_{s}\left(x_{i} \otimes \cdots \otimes x_{i+s-1}\right) \otimes x_{s} \cdots \otimes x_{n}\right) .
\end{array}
$$

An $A_{\infty}$-algebra structure on $V$ is given by a family of maps as in (12) whose family of associators vanishes.

Again, there is a shift involved when we go from the standard definition of differential graded associative algebras to the definition above.

Proposition 3. Let $\left(K, \delta_{0}\right)$ be a complex with cohomology H. Fix a homotopy $h$ of $\delta_{0}$, i.e. a linear map $h$ that satisfies

$$
\delta_{0} \circ h+h \circ \delta_{0}=i d-\Pi_{H} .
$$

From $\Pi_{H}$ one constructs a quasi-isomorphism $\iota: H \rightarrow K$. Let $\delta_{0}+\mathcal{O}(\epsilon)$ be any flat $A_{2}$-algebra structure on $K[[\epsilon]]$.

Then there is a canonical deformation of the trivial $A_{\infty}$-algebra structure on $H[[\epsilon]]$ and a deformation of the quasi-isomorphism $\iota$ that is a quasi-isomorphism between the perturbed $A_{\infty}$-algebra structure on $K[[\epsilon]]$ and the perturbed one on $H[[\epsilon]]$.

We will prove this with the help of the graphical calculus parallel to the discussion given in the previous Subsection. Let $\left(K, D_{\epsilon}, \bullet_{\epsilon}\right)$ be a $A_{\infty}$-algebra structure with only a 1-ary and binary operations (i.e. a flat $A_{2}$-algebra). Assume that $D_{\epsilon}=$ $\delta_{0}+D_{R}$ where $D_{R}$ is $\mathcal{O}(\epsilon)$. Again we construct a representation of $\mathbb{T}_{d}(n)$ on $\operatorname{Hom}_{h}(T(K), K)$. We define

$$
\Psi: \mathbb{T}_{d} \rightarrow \operatorname{Hom}_{h}(T(K), K[1])
$$

by placing the product $\bullet_{\epsilon}$ at every trivalent vertex and insert an appropriate number of $-D_{R}$ 's along all the decorated edges. In between this operations we insert a homotopy $h$ of $\delta_{0}$. Then we define

$$
\Theta: \mathbb{T}_{d} \rightarrow \operatorname{Hom}_{h}(T(K), K[1])
$$

by dividing $\Psi$ by $|A u t([F])| 2$ and

$$
\begin{aligned}
& \widetilde{Z_{n}}: \quad T(K) \rightarrow K[1] \\
& \widetilde{Z_{n}}:=\sum_{G \in \mathbb{T}_{d}(n)} \Theta(G)
\end{aligned}
$$

\footnotetext{
${ }^{2}$ Our definition of orientation implies that the following abstract graphs are different as oriented graphs:
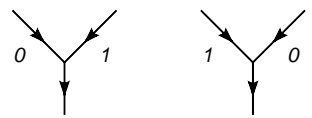

However, if we embed them in the plane, they are equal as oriented planar trees. Given any oriented planar tree $\mathbb{T}$ there are $|\operatorname{Aut}([\mathbb{T}])|$ oriented (in the sense of our definition) abstract trees that map to the given oriented planar tree. Instead of considering the class of graphs we are interested in and later dividing out the automorphism group we could instead just work with the class of oriented planar trees.
} 
and denote the embeddings of these maps by $Z_{n}$. Parallel to Theorem 2 we state

Theorem 3. The family of maps $\left(Z_{n}: T(H) \rightarrow H[1]\right)_{n \geq 1}$ defines an $A_{\infty}$-algebra structure on $H$ that is quasi-isomorphic to the one on $\left(K,-D_{\epsilon}, \bullet_{\epsilon}\right)$. The quasiisomorphism is given explicitly by the family of maps $\left(\iota+h \circ \widetilde{Z_{1}}, h \circ \widetilde{Z}_{2}, h \circ \widetilde{Z}_{3}, \cdots\right)$.

The proof of Theorem 3 is essentially the same as the proof of Theorem 2 .

The next natural question is whether this method to derive higher homotopy structures on cohomology is well-behaved with respect to symmetrization. Let $\left(K, D_{\epsilon}, \bullet_{\epsilon}\right)$ be a flat $A_{2}$-algebra and let $\left(K, D_{\epsilon},[\cdot, \cdot]_{\epsilon}\right)$ denote the symmetrization of this structure, i.e.

$$
[a, b]_{\epsilon}:=a \bullet_{\epsilon} b+(-1)^{|a||b|} b \bullet_{\epsilon} a .
$$

One can do two things: one possibility is to induce an $A_{\infty}$-algebra structure on cohomology first and then symmetrize this new structure, the second one is to induce an $L_{\infty}$-algebra structure on cohomology from the $L_{2}$-algebra structure we obtained by symmetrization. We claim that these two procedures yield the same results, so the method to induce higher homotopy structures on cohomology commutes with symmetrization. The proof is easy:

$$
\begin{aligned}
\sum_{\sigma \in \Sigma_{n}} \operatorname{sign}(\sigma) \sigma^{*} \widetilde{Z}_{n} & =\sum_{\sigma \in \Sigma_{n}} \sum_{F \in \mathbb{T}_{d}(n)} \operatorname{sign}(\sigma) \frac{1}{|\operatorname{Aut}([F])|} \sigma^{*} \Psi(F) \\
& =\sum_{\sigma \in \Sigma_{n}} \sum_{F \in\left[\mathbb{T}_{d}(n)\right]} \operatorname{sign}(\sigma) \frac{1}{|\operatorname{Aut}([F])|} \sigma^{*} \Phi(F) \\
& =\widetilde{M_{n}}
\end{aligned}
$$

and when we compose this identity with $\Pi_{H}$ from the left we obtain the claim. We remark that the equality between the second and the third sum follows directly from the definition of $[\cdot, \cdot]_{\epsilon}$.

\section{Formal DEFORMATIONS OF COISOTROPIC SUBMANIFOLDS}

3.1. The BFV-complex and Lie algebroids - a short review. We want to apply the machinery presented in Section 2 to a problem in Poisson geometry. So we will first give a short review of the facts that will be of importance later.

The setting is the following: let $M$ be a smooth manifold equipped with a Poisson bivector field $\Pi$. We denote the corresponding Poisson bracket on $\mathcal{C}^{\infty}(M)$ by $\{\cdot, \cdot\}_{M}$. To every Poisson manifold one can associate a so-called differential graded symplectic manifold $T^{*}[1] M$. The graded symplectic form on $T^{*}[1] M$ is the canonical graded symplectic form on any shifted cotangent bundle. In local coordinates $x^{i}$ on $M$ and coordinates $p_{i}$ along the fibres of $T^{*}[1] M$ the Poisson bracket of the graded symplectic form is given by

$$
[F, G]=F \frac{\overleftarrow{\partial}}{\partial x^{i}} \frac{\vec{\partial}}{\partial p_{i}} G-F \frac{\overleftarrow{\partial}}{\partial p_{i}} \frac{\vec{\partial}}{\partial x^{i}} G
$$

We remark that $[\Pi, \Pi]=0$ is equivalent to require $\Pi$ to be Poisson. Hence $X_{H}:=[\Pi, \cdot]$ is a cohomological vector field on $T^{*}[1] M$, i.e. a differential of degree +1 on $\mathcal{C}^{\infty}\left(T^{*}[1] M\right)=\Gamma(\bigwedge T M)$. 
One can associate a bundle map $\Pi^{\sharp}: T^{*} M \rightarrow T M$ to $\Pi$ by defining $\Pi^{\sharp}(\alpha)(\beta):=<$ $\Pi, \alpha \wedge \beta>$ (here $\langle\cdot, \cdot\rangle$ denotes contraction). A submanifold $C$ of $M$ is called coisotropic if $\Pi^{\sharp}$ maps the conormal bundle $N^{*} C$ of $C$ into $T C$. Another equivalent definition is to require the vanishing ideal

$$
I(C):=\left\{f \in \mathcal{C}^{\infty}(M) \mid \text { the pullback of } f \text { under } C \hookrightarrow M \text { vanishes }\right\}
$$

of $C$ to be a coisotrope of $\left(\mathcal{C}^{\infty}(M),\{\cdot, \cdot\}_{M}\right)$, i.e. a multiplicative ideal and a Lie subalgebra.

The space of sections of the conormal bundle $N^{*} C$ possesses a natural Lie algebra structure. One observes that $f \in I(C)$ implies $d f \in \Gamma\left(N^{*} C\right)$ and sections of such type locally span $N^{*} C$ at every point of $C$. So the Lie algebra structure on $\Gamma\left(N^{*} C\right)$ is uniquely defined by setting

$$
\begin{gathered}
{[d f, d g]=d\{f, g\}, f, g \in I(C),} \\
{[f \alpha, \beta]=f[\alpha, \beta]-\Pi^{\sharp}(\beta)(f) \alpha, \alpha, \beta \in \Gamma\left(N^{*} C\right), f \in \mathcal{C}^{\infty}(C) .}
\end{gathered}
$$

By construction of the bracket on $\Gamma\left(N^{*} C\right), \Pi^{\sharp}$ induces a Lie algebra homomorphism between $\Gamma\left(N^{*} C\right)$ and $\Gamma(T C)$ (the Lie algebra of vector fields on $C$ ). By definition, this implies that $N^{*} C$ is a Lie algebroid over $C$.

One can construct a differential $\delta$ on $\Gamma(\bigwedge N C)$ which is called the Lie algebroid differential of $C$. For $f \in \mathcal{C}^{\infty}(C)$ one defines

$$
\delta(f):=\Pi^{\sharp}(d \tilde{f}) \bmod T C
$$

where $\tilde{f}$ is any extension of $f$ to a function on all of $M$. The class of $\delta(f)$ modulo $T C$ is well-defined. Take $X \in \Gamma(N C)$ and choose an extension $\tilde{X}$ of $X$ to a vector field on $M$. One defines

$$
\delta(X):=[\Pi, \tilde{X}] \bmod T C
$$

and observes that this is well-defined. Then one extends $\delta$ as a graded derivation to all of $\Gamma(\bigwedge N C)$. The Lie algebroid cohomology of $C$ is the cohomology of $(\Gamma(\bigwedge N C), \delta)$.

One can always find an embedding $N C \hookrightarrow M$ with the help of a tubular neighbourhood of $N$ in $M$. We fix such an embedding once and for all. By this, $N C$ inherits the structure of a Poisson manifold. We denote the Poisson bracket on $\mathcal{C}^{\infty}(N C)$ also by $\{\cdot, \cdot\}_{M}$ and the Poisson bivector field by $\Pi$.

In $\mathrm{OP}$ an $L_{\infty}$-algebra structure on $\Gamma(\bigwedge N C) \cong \mathcal{C}^{\infty}\left(N^{*}[1] C\right)$ was constructed. We will follow the presentation given in $[\mathrm{CF}$. The construction starts with the Poisson bivector field on $N C \hookrightarrow M$ which can be seen as a Maurer-Cartan element of the graded Lie algebra $\mathcal{V}(N C)=\mathcal{C}^{\infty}\left(T^{*}[1] N C\right)$ of multivector fields on $N C$. Using a result of Roytenberg Roy it follows that there is a natural antisymplectomorphism of graded symplectic manifolds

$$
T^{*}[1] N C \rightarrow T^{*}[1] N^{*}[1] C .
$$

This implies that there is an anti-Poisson map

$$
\mathcal{C}^{\infty}\left(T^{*}[1] N C\right) \rightarrow \mathcal{C}^{\infty}\left(T^{*}[1] N^{*}[1] C\right)
$$

The image of $\Pi$ under this map is a Maurer-Cartan element in $\mathcal{C}^{\infty}\left(T^{*}[1] N^{*}[1] C\right)$ which we denote by $P$. Given a Maurer-Cartan element and using the projection 
$\mathcal{P}: \mathcal{V}\left(N^{*}[1] C\right) \rightarrow \mathcal{C}^{\infty}\left(N^{*}[1] C\right)$ one can construct the so-called derived brackets using a method due to Th. Voronov $([\mathrm{V}])$. The structure maps are given by

$$
\begin{aligned}
\lambda_{n}: \mathcal{C}^{\infty}\left(N^{*}[1] C\right)[1] \otimes \cdots \otimes \mathcal{C}^{\infty}\left(N^{*}[1] C\right)[1] & \rightarrow \mathcal{C}^{\infty}\left(N^{*}[1] C\right)[1] \\
f_{1} \otimes \cdots \otimes f_{n} & \mapsto \mathcal{P}\left(\left[\left[\cdots\left[\left[P, f_{1}\right], f_{2}\right] \cdots\right], f_{n}\right]\right) .
\end{aligned}
$$

From the general results contained in $[\mathrm{V}]$ it follows immediately that this family of maps forms the structure of an $L_{\infty}$-algebra on $\mathcal{C}^{\infty}\left(N^{*}[1] C\right)[1]$. Applying the décalage-isomorphism yields an $L_{\infty}$-algebra on $\mathcal{C}^{\infty}\left(N^{*}[1] C\right)$ in the sense of Stasheff's original definition. Moreover it is easy to check that the structure maps of this $L_{\infty}$-algebra are graded multiderivations. In $\left[\mathrm{CF}\right.$ the term $P_{\infty}$-algebra was introduced for such $L_{\infty}$-algebra structures. With the help of this $P_{\infty}$-algebra structure one can state a formal deformation problem associated to any coisotropic submanifold of an arbitrary Poisson manifold, see $\mathrm{OP}$.

We want to remark that the flatness of the $L_{\infty}$-algebra on $\mathcal{C}^{\infty}\left(N^{*}[1] C\right)$ is equivalent to $C$ being coisotropic. Flatness implies that the first structure map is a differential, and it can be checked to be exactly the Lie algebroid differential of $C$. So the Lie algebroid cohomology carries the structure of a graded Poisson algebra and this structure is independent from the embedding $N C \hookrightarrow M$.

There is another construction of a graded Poisson bracket on the Lie algebroid cohomology of $C$ using the Batalin-Vilkovisky-Fradkin complex (BFV for short) that we will introduce now 3 We follow the expositions [CFL and $\mathrm{St}$. We define $\mathcal{M}$ to be the graded manifold

$$
\mathcal{M}:=N C \oplus N^{*}[1] C \oplus N[-1] C
$$

and introduce local coordinates for convenience: $x^{i}$ denote local coordinates on $C, y^{\mu}$ are linear coordinates along the fibres of $N C, b^{\mu}$ linear coordinates along the fibres of $N[-1] C$ and $c_{\mu}$ linear coordinates along the fibres of $N^{*}[1] C$. In the physical literature the $c_{\mu}$ 's are referred to as the ghosts and $b^{\mu}$ 's are called the ghost-momenta. The space is bi-graded by the ghost-degree and the ghostmomentum degree (which is minus the homogeneous degree in the ghost-momentum variables). The sum of them both is defined to be the total degree. There is a natural differential on $K:=\mathcal{C}^{\infty}(\mathcal{M})$ defined by

$$
\delta_{0}:=y^{\mu} \frac{\partial}{\partial b^{\mu}}
$$

which is called the Koszul-differential. It is globally well-defined and one can prove that

$$
H\left(K, \delta_{0}\right)=\mathcal{C}^{\infty}\left(N^{*}[1] C\right) .
$$

Hence $\left(K, \delta_{0}\right)$ is a resolution of $\mathcal{C}^{\infty}\left(N^{*}[1] C\right)$ in the sense of homological algebra. There is a natural homotopy for $\delta_{0}$ which is given by

$$
\widetilde{h}:=b^{\mu} \frac{\partial}{\partial y^{\mu}} .
$$

\footnotetext{
${ }^{3} \mathrm{We}$ remark that there is a connection between the BFV-complex and the BV formalism that we outlined in Subsection 2.1 This connection corresponds to the transition from the Hamiltonian to the Lagrangian setting of classical mechanics (or classical field theory).
} 
It is not hard to see that

$$
\delta_{0} \circ \widetilde{h}+\widetilde{h} \circ \delta_{0}=y^{\mu} \frac{\partial}{\partial y^{\mu}}+b^{\mu} \frac{\partial}{\partial b^{\mu}}
$$

and to obtain a homotopy that satisfies equation (7) we should normalize $\widetilde{h}$ to

$$
h(f):=\frac{1}{\mid \text { ghost-momentum deg. of } f|+| \text { deg. of } f \text { in the normal coordinates } y \mid} \widetilde{h}(f)
$$

whenever the denominator does not vanish and 0 otherwise.

Next, we want to equip $K$ with the structure of a graded Poisson algebra. This works under the assumption that $N C$ is a flat vector bundle over $C$ (how to avoid this assumption is explained in Appendix E). In this case, there is the graded Poisson structure given by the pairing between ghosts and ghost-momenta. One defines

$$
\left\{c_{\mu}, b^{\lambda}\right\}_{g}:=\delta_{\mu}^{\lambda}
$$

and extends this bracket by graded-skew symmetry and the graded Leibniz rule to all of $\mathcal{C}^{\infty}\left(N^{*}[1] C \oplus N[-1] C\right)$. We have seen that $\mathcal{C}^{\infty}(N C)$ inherits a Poisson structure via the identification with a tubular neighbourhood of $C$ in $M$ which we denoted by $\{\cdot, \cdot\}_{M}$. Locally, the Poisson structure we are interested in is the sum of $\{\cdot, \cdot\}_{g}$ and $\{\cdot, \cdot\}_{M}$. Since $N C$ is flat we can always find a vector bundle atlas whose transition functions are locally constant. If we use one of these atlases, all the local Poisson structures can be glued together.

Observe that $\delta_{0}$ is an inner derivation with respect to $\{\cdot, \cdot\}_{g}$ :

$$
\delta_{0}(\cdot):=\left\{y^{\mu} c_{\mu}, \cdot\right\}_{g} .
$$

But $\delta_{0}$ is not an inner derivation with respect to the total bracket $\{\cdot, \cdot\}:=\{\cdot, \cdot\}_{M}+$ $\{\cdot, \cdot\}_{g}$ and does not satisfy the Leibniz rule with respect to $\{\cdot, \cdot\}$. Hence there is no canonical structure of a graded Poisson algebra on $H\left(K, \delta_{0}\right)=\mathcal{C}^{\infty}\left(N^{*}[1] C\right)$. However, Batalin, Fradkin and Vilkovisky showed that one can find correction terms in negative ghost-momentum degrees to $\Omega_{0}:=y^{\mu} c_{\mu}$ such that the sum $\Omega$ of $\Omega_{0}$ and the correction terms $\Omega_{i}$ satisfies

$$
\{\Omega, \Omega\}=0
$$

and consequently $(K, D:=\{\Omega, \cdot\},\{\cdot, \cdot\})$ is a differential graded Poisson algebra. Stasheff showed that this extension has a mathematical interpretation in terms of homological perturbation theory. The differential complex $(K, D)$ is referred to as the BFV-complex. By construction $H(K, D)$ is a graded Poisson algebra. We decompose $D=\{\Omega, \cdot\}$ into maps that are homogeneous in the ghost degree

$$
D=\sum_{j \geq 0} \delta_{j}
$$

and observe that $\delta_{0}$ is the Koszul-differential. The induced differential on $H\left(K, \delta_{0}\right)=$ $\mathcal{C}^{\infty}\left(N^{*}[1] C\right)$ is simply the map induced from $\delta_{1}$ and this induced differential is the Lie algebroid differential $\delta$. It follows from Corollary 1 that $H(K, D) \cong$ $H\left(H\left(K, \delta_{0}\right), \delta\right)=H(\Gamma(\bigwedge N C), \delta)$. So $H(K, D)$ is isomorphic to the Lie algebroid cohomology and this isomorphism can be used to transfer the graded Poisson bracket from $H(K, D)$ to the Lie algebroid cohomology of $C$. 
Naturally the question arises whether the graded Poisson algebra structures on the Lie algebroid cohomology associated to $C$ constructed in these two very different ways are equivalent (under the assumption that the BFV-complex as we introduced it is defined for $C$ ). We will give an affirmative answer to this question. Moreover we will show that $(K, D,\{\cdot, \cdot\})$ and $\mathcal{C}^{\infty}\left(N^{*}[1] C\right)$ equipped with the $L_{\infty}$-algebra structure we described above are quasi-isomorphic as $L_{\infty}$-algebras. From this the statement about the equivalence of the two graded Poisson algebra structures on the Lie algebroid cohomology immediately follows. A much stronger consequence of the statement is that the two deformation functors associated to $(K, D,\{\cdot, \cdot\})$ and $\mathcal{C}^{\infty}\left(N^{*}[1] C\right)$ (equipped with the $P_{\infty}$-algebra structure of Oh and Park) are equivalent.

3.2. Perturbation theory for the BFV-complex. Next we apply Theorem 2 to the BFV-complex as introduced in the previous Subsection. Throughout this and the next Subsection we always assume that $N C$ is a flat vector bundle (for example this is the case whenever $C$ is defined using global constraints). In Appendix E we present a way to deal with the general situation and show that the local result can be extented in a straight-forward manner.

We start with $\left(K:=\mathcal{C}^{\infty}(\mathcal{M}), \delta_{0}\right)$ and $H=\mathcal{C}^{\infty}\left(N^{*}[1] C\right)$ as the unperturbed structures. The perturbation of $\left(K, \delta_{0}\right)$ is constructed as follows: we start with $\Omega_{0}:=y^{\mu} c_{\mu}$ and want to find a power series $\Omega_{\epsilon}$ in $\epsilon$ such that

$$
\left\{\Omega_{\epsilon}, \Omega_{\epsilon}\right\}_{\epsilon}=0
$$

where $\{\cdot, \cdot\}_{\epsilon}:=\{\cdot, \cdot\}_{g}+\epsilon\{\cdot, \cdot\}_{M}$. The solution of this problem is exactly given by Stasheff's procedure in [St]. The ghost-momentum degree -1 part of $\Omega_{\epsilon}$ is given by

$$
-\frac{\epsilon}{2} \frac{\partial \Pi^{\alpha \beta}}{\partial y^{\mu}} b^{\mu} c_{\alpha} c_{\beta} .
$$

We define $D_{\epsilon}:=\left\{\Omega_{\epsilon}, \cdot\right\}$ and clearly $\left(K[[\epsilon]], D_{\epsilon}, \epsilon\{\cdot, \cdot\}_{\epsilon}\right)$ is a differential graded Lie algebra which is a perturbation of $\delta_{0}$. So we can apply Theorem 2 and obtain an $L_{\infty}$-algebra structure on $H[[\epsilon]]$. We want to give a more detailed description of this structure now.

We forget about the signs involved and make some considerations on the ghostmomentum degree. We start with some arguments in the cohomology $\mathcal{C}^{\infty}\left(N^{*}[1] C\right) \hookrightarrow$ $\bigoplus_{q \geq 0} K^{(q, 0)}$ and in the end we have to project back to the cohomology. So the ghost-momentum degree of the operations that can contribute to the $L_{\infty}$-algebra structure must be zero. $D_{R}=\left\{\epsilon \Omega_{1}+\epsilon^{2} \Omega_{2} \cdots, \cdot\right\}_{\epsilon}$ has inhomogeneous ghostmomentum degree smaller or equal to 0 , the homotopy $h$ has ghost-momentum degree -1 , the bracket $\{\cdot, \cdot\}_{M}$ has ghost-momentum degree 0 and the bracket $\{\cdot, \cdot\}_{g}$ has ghost-momentum degree +1 . So for a map that consists of the two brackets and $D_{R}$ 's with the homotopies in between and that must have ghost-momentum degree zero it follows that only the two following diagrams can possibly contribute: 


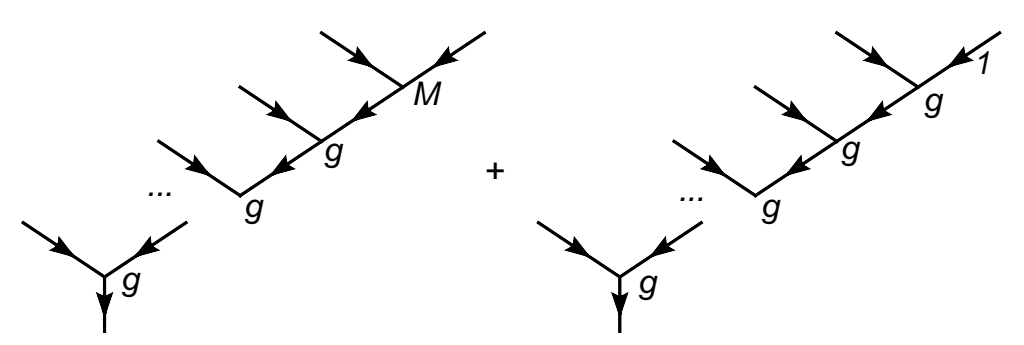

where all the edges without a number are decorated by 0 , and the labels $g$ and $M$ reflect the fact that we apply the bracket coming from the pairing between $N^{*}[1] C$ and $N[-1] C$ or the one coming from the Poisson structure on $M$ (as always the internal edges carry the homotopy $h$ ). Moreover, only the ghost-momentum 0 part $\delta_{1}$ of $D_{R}$ can contribute. Restricted to $H=\mathcal{C}^{\infty}\left(N^{*}[1] C\right)$ it is given by

$$
\delta_{1}=\epsilon\left(c_{\mu} \Pi^{\mu i} \frac{\partial}{\partial x^{i}}-\frac{1}{2} \frac{\partial \Pi^{\alpha \beta}}{\partial y^{\tau}} c_{\alpha} c_{\beta} \frac{\partial}{\partial c_{\tau}}\right)
$$

in local coordinates.

We denote the two diagrams by $\mathcal{R}(n)$ and $\mathcal{S}(n)$ respectively.

More generally one observes that also without projecting to cohomology there is only a finite number of operations when we fix the number of inputs. This is due to the fact that whenever we add a $D_{R}$ we also have to add a homotopy, so the ghostmomenta degree decreases. So there can be only a bounded number of $D_{R}$ 's and this implies that there is only a bounded number of diagrams with a fixed number of inputs that contribute to the $L_{\infty}$-algebra structure and the $L_{\infty}$-morphism we are considering in Theorem 2, So we are allowed to set $\epsilon$ equal to 1.

We denote the two maps associated to $\mathcal{R}(n)$ and $\mathcal{S}(n)$ by

$$
\begin{aligned}
R(n): T^{n}(H) & \rightarrow H[2-n] \text { and } \\
S(n): T^{n}(H) & \rightarrow H[2-n]
\end{aligned}
$$

respectively. What are the signs of $R(n)$ and $S(n)$ ? As said before, we should not have started from $\left(K, D_{\epsilon},\{\cdot, \cdot\}_{\epsilon}\right)$ but from $\left(K,-D_{\epsilon},\{\cdot, \cdot\}_{\epsilon}\right)$. When we do this, we do not have to put $-D_{R}$ along the decorated lines but just $D_{R}$ instead. On the other hand we have to use $-h$ now instead of $h$ as the homotopy. Moreover, when one translates our structures from Stasheff's to Voronov's definition one sees that one should associate $(-1)^{\operatorname{deg}(a)+1}\{a, b\}$ to the trivalent vertices where $\operatorname{deg}(\mathrm{a})$ is the total degree of $a$ in $K$. All this results into

$$
\begin{aligned}
& R(n)\left(x_{1} \otimes \cdots \otimes x_{n}\right)=-\frac{1}{2}(-1)^{\sum_{i=1}^{n-1} \operatorname{deg}\left(x_{i}\right)}\left\{x_{1}, h\left\{x_{2}, \cdots, h\left\{x_{n-1}, x_{n}\right\}_{M}\right\}_{g} \cdots\right\}_{g}, \\
& S(n)\left(x_{1} \otimes \cdots \otimes x_{n}\right)=(-1)^{\sum_{i=1}^{n-1} \operatorname{deg}\left(x_{i}\right)}\left\{x_{1}, h\left\{x_{2}, \cdots, h\left\{x_{n-1}, h \delta_{1}\left(x_{n}\right)\right\}_{g}\right\}_{g} \cdots\right\}_{g} .
\end{aligned}
$$

Observe that we first have to compute the symmetrization of $\Phi(\mathcal{R}(n))+\Phi(\mathcal{S}(n))$ with respect to the degrees in $K[1]$ and then apply the décalage-isomorphism to go to Stasheff's setting.

We claim that the $L_{\infty}$-algebra structure we obtain is even a $P_{\infty}$-algebra structure, i.e. the structure maps are all multiderivations. First we will argue (forgetting the signs for a moment) that when we plug a product $a \cdot b$ in one of the inputs of $R(n)$ we obtain the sum of $a$ times $R(n)$ applied to $b$ and $b$ times $R(n)$ applied to 
$a$. So assume we put $a b$ into one of the inputs of $\{\cdot, \cdot\}_{M}$. There the Leibniz rule applies. When we plug this sum into the homotopy and assume that it is also a derivation with respect to multiplication we observe that only the factors where $h$ is applied to the expression in $\{\cdot, \cdot\}_{M}$ survive. This is because $h$ vanishes on elements that are in $H$. Whenever we take the $g$-bracket of the resulting expression with something in $H$, all terms containing $\{\cdot, a\}_{g}$ or $\{\cdot, b\}_{g}$ vanish because both expressions in the bracket do not contain any ghosts the ghost-momenta could get paired with. Inductively it follows that in the end only the two sums $a \cdot R(n)(\cdots b \cdots)$ and $R(n)(\cdots a \cdots) \cdot b$ remain. The same arguments apply to all the other places where we can plug in the product $a b$ and it is also valid for $R(n)$. Concerning the signs we prove

Proposition 4. Let $\left(\mu_{n}: \bigwedge^{n}(H) \rightarrow H[2-n]\right)_{n \geq 1}$ be the induced $L_{\infty}$-algebra structure on $H=\mathcal{C}^{\infty}\left(N^{*}[1] C\right)$. Then

$$
\begin{array}{r}
\mu_{n}\left(x_{1} \otimes \cdots \otimes x_{n-1} \otimes a b\right)=\mu_{n}\left(x_{1} \otimes \cdots \otimes x_{n-1} \otimes a\right) b \\
+(-1)^{\operatorname{deg}(a)\left(\operatorname{deg}\left(x_{1}\right)+\cdots \operatorname{deg}\left(x_{n-1}\right)+n-2\right)} a \mu_{n}\left(x_{1} \otimes \cdots \otimes x_{n-1} \otimes b\right)
\end{array}
$$

i.e. $\left(\mu_{n}: \bigwedge^{n}(H) \rightarrow H[2-n]\right)_{n \geq 1}$ form a $P_{\infty}$-algebra structure.

Proof. First one computes the outcome of $R(n)$ for $x_{1} \otimes \cdots \otimes x_{r} \otimes a b \otimes x_{r+1} \cdots \otimes x_{n-1}$ which gives

$$
\begin{aligned}
& R(n)\left(x_{1} \otimes \cdots \otimes x_{r} \otimes a b \otimes x_{r+} \otimes \cdots \otimes x_{n-1}\right)= \\
& (-1)^{\operatorname{deg}(a)\left(\operatorname{deg}\left(x_{1}\right)+\cdots \operatorname{deg}\left(x_{r}\right)+r+1\right)} a R(n)\left(x_{1} \otimes \cdots \otimes x_{r} \otimes b \otimes x_{r+1} \otimes \cdots \otimes x_{n-1}\right)+ \\
& (-1)^{\operatorname{deg}(b)\left(\operatorname{deg}\left(x_{r+1}\right)+\cdots \operatorname{deg}\left(x_{n}\right)+n-r-1\right)} R(n)\left(x_{1} \otimes \cdots \otimes x_{r} \otimes a \otimes x_{r+1} \otimes \cdots \otimes x_{n-1}\right) b
\end{aligned}
$$

and the same equation holds for $S(n)$ too. If we add the Koszul-sign induced by the representation of the permutation that shifts $a b$ to the $r+1$ 'th position we have to multiply the last two lines by

$$
\begin{aligned}
& (-1)^{\operatorname{deg}(a)\left(\operatorname{deg}\left(x_{r+1}\right)+\cdots+\operatorname{deg}\left(x_{n-1}\right)+n-r-1\right)} \text { and } \\
& (-1)^{\operatorname{deg}(b)\left(\operatorname{deg}\left(x_{r+1}\right)+\cdots+\operatorname{deg}\left(x_{n-1}\right)+n-r-1\right)}
\end{aligned}
$$

respectively and the claim follows immediately.

Now we want to compare the induced $P_{\infty}$-algebra structure on $H=\mathcal{C}^{\infty}\left(N^{*}[1] C\right)$ with the one constructed in $\mathrm{OP}$. Let $x^{i}$ be local coordinates on $C, p_{i}$ coordinates along the fibres of $T^{*}[1] C, c_{\mu}$ coordinates along the fibres of $N^{*}[1] C$ and $y^{\mu}$ coordinates along the fibres of $T^{*}[1] N^{*}[1] C$. The Poisson bracket is given by

$$
[\cdot, \cdot]=\left(\frac{\overleftarrow{\partial}}{\partial x^{i}} \frac{\vec{\partial}}{\partial p_{i}}-\frac{\overleftarrow{\partial}}{\partial p_{i}} \frac{\vec{\partial}}{\partial x^{i}}\right)+\left(\frac{\overleftarrow{\partial}}{\partial c_{\mu}} \frac{\vec{\partial}}{\partial y^{\mu}}-\frac{\overleftarrow{\partial}}{\partial y^{\mu}} \frac{\vec{\partial}}{\partial c_{\mu}}\right)
$$

Define the structure maps $\tau_{n}$ of the $P_{\infty}$-algebra to be the structure maps corresponding to

$$
\left(f_{1} \otimes \cdots \otimes f_{n}\right) \mapsto \mathcal{P}\left[\left[\cdots\left[\left[P, f_{1}\right], f_{2}\right] \cdots\right], f_{n}\right]
$$

under the décalage-isomorphism. Here $P$ is the image of the Poisson bivector field $\Pi$ under the identification of the shifted cotangent bundles of $N C$ and $N^{*}[1] C$. One 
obtains

$$
\begin{aligned}
\tau_{n}\left(c_{\nu_{1}} \otimes \cdots \otimes c_{\nu_{(n-2)}} \otimes f \otimes g\right) & =\left.(-1)^{n}\left(\frac{\partial^{(n-2)}}{\partial y^{\nu_{1}} \cdots y^{\nu_{(n-2)}}}\{f, g\}\right)\right|_{C} \\
\tau_{n}\left(c_{\nu_{1}} \otimes \cdots \otimes c_{\nu_{(n-1)}} \otimes f\right) & =\left.(-1)^{n}\left(\frac{\partial^{(n-1)}}{\partial y^{\nu_{1}} \cdots y^{\nu_{(n-1)}}}\{f, \cdot\}\right)\right|_{C} \\
\tau_{n}\left(c_{\nu_{1}} \otimes \cdots \otimes c_{\nu_{n}}\right) & =\left.(-1)^{n}\left(\frac{\partial^{n}}{\partial y^{\nu_{1}} \cdots y^{\nu_{n}}}\{\cdot, \cdot\}\right)\right|_{C}
\end{aligned}
$$

where $f$ and $g$ are just functions on $C$, i.e. they only depend on the $x$-coordinates. The free slots marked by - mean that we interpret the last two expressions on the right hand side as linear operators on $\Gamma\left(N^{*} C\right)$ and $\Gamma\left(N^{*} C\right) \otimes \Gamma\left(N^{*} C\right)$ respectively.

We compute the results for the same type of arguments when we plug them into $\mu_{n}$. For elements that contain two functions on $C$ only the diagram $\mathcal{R}(n)$ contributes and we are forced to place these two functions on the inputs of $\{\cdot, \cdot\}_{M}$, so we obtain $\{f, g\}_{M}$. Next we have to apply the homotopy $h$ and obtain

$$
\frac{1}{(n-2)} b^{\nu} \frac{\partial \Pi^{i j}}{\partial y^{\nu}} \frac{\partial f}{\partial x^{i}} \frac{\partial g}{\partial x^{j}}
$$

(the factor $1 /(n-2)$ comes from the fact that we need to normalize the homotopy) and applying $\left\{c_{\nu_{(n-2)}}, \cdot\right\}_{g}$ results into forgetting the $b$ and replacing $y^{\nu}$ by $y^{\nu_{(n-2)}}$. When we take care of the signs in $R(n)$ and the ones coming from the décalageisomorphism we obtain

$$
(-1)^{(n-2)} \frac{1}{2(n-2) !} \frac{\partial^{(n-2)}}{\partial y^{\nu_{1}} \cdots y^{\nu_{(n-2)}}}\left(\{f, g\}_{C}\right) \text {. }
$$

The factor $1 /(2(n-2)$ !) disappears if we sum over all permutations among the ghosts and among $f$ and $g$.

For the element only consisting of ghosts and one function on $C$ only the diagram $\mathcal{S}(n)$ contributes and we are forced to put $f$ into the last slot and again an easy computation shows that the result is exactly the expression we get when we apply $\tau_{n}$. For an element of the exterior algebra that only consists of ghosts only the diagram $\mathcal{S}(n)$ contributes and again we obtain the same result as for $\tau_{n}$.

Since $\left(\tau_{n}: \bigwedge^{n}(H) \rightarrow H[2-n]\right)_{n \geq 2}$ and $\left(\mu_{n}: \bigwedge^{n}(H) \rightarrow H[2-n]\right)_{n \geq 2}$ are both $P_{\infty}$-algebra structure they are totally determined by their values on these three special classes of elements, see [CF]. Hence

Theorem 4. Let $C$ be a coisotropic submanifold of $M$. Then the induced $P_{\infty}$ algebra structure on $\Gamma(\bigwedge N C)$ coincides with the $P_{\infty}$-algebra constructed by $O h$ and Park. Consequently, the last one is quasi-isomorphic to the BFV-complex regarded as a differential graded Lie algebra. Moreover it follows that the formal deformation functors associated to the BFV-complex and to the $P_{\infty}$-algebra structure of $O h$ and Park are equivalent.

Strictly speaking, we proved Theorem 4 only under the assumption that the normal bundle to $\mathrm{C}$ is flat so far. We present a globalisation of the BFV-complex in the non-flat case which is due to M. Bordemann and H.-C. Herbig, (see [B] and $[\mathrm{H}]$ and references therein) and show that Theorem 4 extends to this globalisation in Appendix $\mathrm{E}$. 
Next we give a presentation of the formal deformation problem that is associated to the $\mathrm{BFV}$-complex, establish a connection to the geometric deformation problem and investigate an example that first appeared in [Z].

3.3. The deformation problem and the BFV-complex. The deformation problem for the BFV-complex has the following form: $F \in \epsilon K^{1}[[\epsilon]]$ is a MaurerCartan element iff

$$
\{\Omega+F, \Omega+F\}=0
$$

i.e. $F$ is a formal deformation of $\Omega$ such that the perturbation does not violate $\{\Omega, \Omega\}=0$. Moreover two elements $F$ and $G$ in $\epsilon K^{1}[[\epsilon]]$ are gauge-equivalent iff there is an element $B \in \epsilon K^{0}[[\epsilon]]$ such that

$$
\Omega+G=e^{\operatorname{ad}_{B}}(\Omega+F)
$$

i.e. $\Omega+G$ and $\Omega+F$ can be connected by the flow of a (formal) Hamiltonian vector field. Using homological perturbation theory if follows that when the ghostmomentum 0 part of a Maurer Cartan element $F$ vanishes, it can be gauged to 0 . So the possibly non-trivial deformations of $\Omega$ are those with non-vanishing ghostmomentum 0 part.

Oh and Park proved that any element that defines a cohomology class in the Lie algebroid cohomology associated to the coisotropic submanifold can be extended to a Maurer-Cartan element if one assumes that the second Lie algebroid cohomology vanishes, see $[\mathrm{OP}$. One can easily recover this fact from the description in terms of the BFV-complex: assume $a_{1} \in K^{1}$ satisfies $D\left(a_{1}\right)=0(D=\{\Omega, \cdot\})$, hence $\left[a_{1}\right] \in H^{1}(K, D) \cong H^{1}\left(H\left(K, \delta_{0}\right),\left[\delta_{1}\right]\right)$. In order to extend $a_{1}$ to a Maurer-Cartan element $a=\sum_{k \geq 1} \epsilon^{k} a_{k}$ one has to solve the following family of equations:

$$
D\left(a_{k}\right)=-\frac{1}{2} \sum_{r+s=k}\left\{a_{r}, a_{s}\right\}
$$

iteratively. If one assumes that $H^{2}(K, D)\left(\cong H^{2}\left(H\left(K, \delta_{0}\right),\left[\delta_{1}\right]\right)\right)=0$ then there are no obstructions (that the right hand side is $D$-closed at every step follows by induction from (19) for all orders $<k$ and from the Leibniz-rule and Jacobi-identity).

Moreover, it follows that vanishing of the first Lie algebroid cohomology implies formal algebraic rigidity:

Lemma 4. Suppose that the first Lie algebroid cohomology associated to a coisotropic submanifold $C$ vanishes. Then $C$ is formally rigid, i.e. it admits no non-trivial formal deformations (as a coisotropic submanifold).

It is a general fact that vanishing of the second cohomology implies unobstructedness of the formal deformation problem and vanishing of the first cohomology implies rigidity. Since the cohomology of the BFV-complex and the Lie algebroid cohomology are isomorphic the Lemma follows directly from the general statement.

We remark that formal deformations that are equivalent to the trivial MaurerCartan element in the $L_{\infty}$-algebra discovered by $\mathrm{Oh}$ and Park need not be trivial in the geometric sense - there might be essentially more 'algebraic' equivalences than 'geometric' one (see the remarks after the prove of Proposition 5). This is why we 
speak of 'algebraic rigidity'.

What is the geometric content of equation (18)? First we observe that sections of $N C$ can be interpreted as elements of degree one in $\mathcal{C}^{\infty}\left(N^{*}[1] C\right)$ and are thus naturally contained in the BFV-complex as elements of ghost degree 1 and ghostmomentum degree 0 . In general elements of this bidegree are of the form $c_{\mu} g^{\mu}(x, y)$ for some functions $g^{\mu}$ depending both on the coordinates on $C$ and fibre coordinates on $N C$. We call elements for which these functions only depend on the coordinates on $C$ geometric. Moreover, every element of the BFV-complex of total degree 1 has a component of ghost degree 1 and ghost-momentum degree 0 . If this component is geometric, we say that the total element is geometric. In particular we can speak of geometric Maurer-Cartan elements. Assume that $c_{\mu} f^{\mu}$ is geometric, i.e. it can be interpreted as a section of the normal bundle of $C$. We can use this element to define new 'constraints'

$$
\phi^{\mu}:=y^{\mu}+f^{\mu}
$$

and a new submanifold given by setting $\phi^{\mu} \equiv 0$ for all $\mu$. The question arises under which conditions this submanifold is coisotropic. The next Proposition shows that this question is directly connected to the Maurer-Cartan equation (18).

Proposition 5. Given a formal section $c_{\mu} f^{\mu}$ of the normal bundle of $C$, the submanifold defined by the constraints (20) is coisotropic iff $c_{\mu} f^{\mu}$ can be extended to a Maurer-Cartan element $F$ of the BFV-complex by adding terms of negative ghostmomentum degree and all such possible extensions are gauge-equivalent. Given any Maurer-Cartan element of the BFV-complex there is a gauge-equivalent MaurerCartan element that is geometric.

Proof. We start by considering the following map

$$
\delta_{f}:=\left\{\Omega_{0}+c_{\mu} f^{\mu}, \cdot\right\}_{g}
$$

which clearly is a differential and can be considered as a perturbation of $\delta_{0}$. From Lemma2 2 one immediately obtains that the cohomology of $\delta_{f}$ is equal to $\mathcal{C}^{\infty}\left(N^{*}[1] C\right)$. Inspecting explicit formulas from GL for the perturbed homotopy and the perturbed projection to cohomology one sees that 1.) all elements of negative ghostmomentum degree project to zero under the perturbed projection and 2.) the perturbed homotopy decreases the ghost-momentum degree by 1 . If we set $\epsilon=0$, $\delta_{f}$ reduces to $\delta_{0}$ (since $c_{\mu} f^{\mu}$ starts at order $\epsilon$ ) we obtain the ordinary projection and the homotopy (13). Following Stasheff's arguments in St one observes that $\Omega_{0}+c_{\mu} f^{\mu}=c_{\mu}\left(y^{\mu}+f^{\mu}\right)$ can be extended to a Maurer-Cartan element iff

$$
\left\{c_{\mu}\left(y^{\mu}+f^{\mu}\right), c_{\gamma}\left(y^{\gamma}+f^{\gamma}\right)\right\}_{M}=c_{\mu} c_{\gamma}\left\{y^{\mu}+f^{\mu}, y^{\gamma}+f^{\gamma}\right\}_{M}
$$

is $\delta_{f}$-exact. But this is the case exactly if there are some functions $h_{\alpha}^{\mu \gamma}$ such that (21) is equal to

$$
\delta_{f}\left(c_{\mu} c_{\gamma} b^{\alpha} h_{\alpha}^{\mu \gamma}\right)=c_{\mu} c_{\gamma} h_{\alpha}^{\mu \gamma}\left(y^{\alpha}+f^{\alpha}\right)
$$

This means that

$$
\left\{\phi^{\mu}, \phi^{\gamma}\right\}_{M}=h_{\alpha}^{\mu \gamma} \phi^{\alpha}
$$

so the vanishing ideal of the submanifold defined by $\phi^{\mu}$ is a Lie subalgebra of $\left(\mathcal{C}^{\infty}(N C),\{\cdot, \cdot\}_{M}\right)$ and hence this submanifold is coisotropic.

Moreover one can always choose the extension to be the $\Omega$ constructed by Stasheff 
at order $\epsilon=0$. Adapting arguments from $[\mathrm{St}$ it follows that the extension that differ at higher orders in $\epsilon$ are all (formally) gauge-equivalent.

That all Maurer-Cartan elements can be gauged to geometric ones also follows from homological perturbation theory: Consider the $y$-dependent part of $c_{\mu} f^{\mu}$ that is of lowest order in $\epsilon$, which we denote by $c_{\mu} g^{\mu}$. Clearly it is $\delta_{0}$-closed and its cohomology class is 0 . If we act by the gauge-transformation

$$
e^{a d\left(\tilde{h}\left(c_{\mu} g^{\mu}\right)\right)}
$$

where $\tilde{h}$ is the homotopy for $\delta_{0}$, we kill $c_{\mu} g^{\mu}$. Proceeding over orders in $\epsilon$ shows that we arrive at a gauge-equivalent element that is geometric in the end.

Propsotion 5 essentially says that there is a correspondence of formal deformations of $C$ that are again coisotropic and Maurer-Cartan elements of the BFVcomplex up to certain gauge-equivalences. The next step is to understand if there is a correspondence between flows of formal Hamiltonian functions and the gaugeequivalences that act on the set of Maurer-Cartan elements of the BFV-complex. Assume we start with two formal sections $c_{\mu} f^{\mu}$ and $c_{\mu} g^{\mu}$ of the normal bundle of $C$. We say that there is a geometric equivalence between these two elements if there exists a formal function $B$ whose Hamiltonian flow maps $y^{\mu}+f^{\mu}$ to $y^{\mu}+g^{\mu}$. Observe that $B$ might depend on the coordinates transversal to $C$, the only important point is that its flow maps the element $y^{\mu}+f^{\mu}$ to $y^{\mu}+g^{\mu}$ where $f^{\mu}$ and $g^{\mu}$ depend only on the coordinates along $C$. It is easy to see that such a geometric equivalence lifts to an equivalence between two Maurer-Cartan elements corresponding to $c_{\mu} f^{\mu}$ and $c_{\mu} g^{\mu}$ : start with any geometric Maurer-Cartan element whose ghostmomentum 0 part is $c_{\mu} f^{\mu}$ and act on it by $e^{a d(B)}$. Since $B$ is of ghost degree 0 and ghost-momentum degree 0 it follows that $e^{a d(B)}\left(c_{\mu}\left(y^{\mu}+f^{\mu}\right)\right)$ is a Maurer-Cartan element that extends $g^{\mu}$. Hence we proved that all formal sections that define coisotropic submanifolds and that are geometrically equivalent posses extensions to Maurer-Cartan elements that are gauge-equivalent in the BFV-complex.

On the other hand one should prove that all geometric Maurer-Cartan elements of the BFV-complex which are gauge-equivalent are 'geometrically' gaugeequivalent. The point is that the BFV-complex contains more elements of total degree 0 than elements whose ghost degree are 0 and whose ghost-momentum degree are 0 . However, only the part with ghost-momentum degree 0 and ghostmomentum degree -1 affects the ghost degree +1 part of the Maurer-Cartan elements. The problem is that actions which come from an element that also contains a non-vanishing ghost-momentum degree -1 part have no geometric meaning. Consequently we call a gauge-equivalence between two geometric Maurer-Cartan elements geometric if its Hamiltonian does not contain a part of ghost degree 1 and ghostmomentum degree -1 . We were not able to show that one can get rid of the nongeometric gauge-equivalences with the help of homological perturbation theory or that they are not allowed since they must map a geometric Maurer-Cartan element to another geometric one. If there were two geometric Maurer-Cartan elements that are gauge-equivalent but for which there is no geometric gauge-equivalence, the quotient space of Maurer-Cartan elements modulo gauge-equivalences would be 'smaller' than expected. On the other hand if one could show that all gaugeequivalences between two geometric Maurer-Cartan elements can be reduced to geometric ones the quotient space would be in one-to-one correspondence with the 
space of formal deformations of $C$ that are coisotropic modulo formal Hamiltonian equivalences.

Although we are not able to present a solution (or counterexample) we want to outline some ideas about how one could at least try to understand the situation. Let us consider a simpler problem similar to ours in the setting of a differential complex with differential $d$. Maurer-Cartan elements are elements of degree 1 that are $d$-closed. There is an action of elements of degree 0 on the set of Maurer-Cartan element. Let $y$ be of degree 0 , it acts by mapping $x$ to $x+d y$. Assume that the action of $y$ on the complex maps $x$ to $x^{\prime}$. We can ask whether there is an element $y^{\prime}$ of a special form that also maps $x$ to $x^{\prime}$. For sure one can change $y$ to $y+d z$ without changing source and target of the transformation given by adding $d y$. If we can find a $z$ such that $y+d z$ has the special form, we are done. This easy example can be generalized to arbitrary $L_{\infty}$-algebra structures on spaces which contain elements of negative degree. The BFV-complex is such a space (except in the trivial case where $M=C)$. What is essential here is that there is an action of elements of degree -1 on gauge-equivalences. To be more precise we fix two Maurer-Cartan elements $F$ and $G$ and denote the space of all gauge-equivalences that map $F$ to $G$ by $\mathcal{G}(F, G)$. The action of $\epsilon K^{-1}[[\epsilon]]$ on $\mathcal{G}(F, G)$ is given by

$$
\begin{aligned}
\epsilon K^{-1}[[\epsilon]] \times \mathcal{G}(F, G) & \rightarrow \mathcal{G}(F, G) \\
\left(z, e^{\operatorname{ad}(B)}\right) & \mapsto e^{\operatorname{ad}(B)} e^{\operatorname{ad}(\{\Omega+F, z\}) .}
\end{aligned}
$$

It is clear that this action preserves $\mathcal{G}(F, G)$. With the help of this action one can construct a 2-groupoid over the set of Maurer-Cartan elements as explained in [G]. The new gauge-equivalence is generated by the Hamiltonian $B C H(B,\{\Omega+F, z\})$, i.e. the Baker-Campbell-Hausdorff formula applied to $B$ and $\{\Omega+F, z\}$. Its lowest order terms are

$$
B C H(B,\{\Omega+F, z\})=B+\{\Omega+F, z\}+\frac{1}{2}\{B,\{\Omega+F, z\}\}+\ldots
$$

which can be used to try to kill the part of $B$ in ghost degree +1 and ghostmomentum degree -1 . However, we could not find convincing arguments that this can always be done.

Finally we consider an example that was first presented in $\mathbf{Z}$ and that was also investigated in $\mathrm{OP}$. M. Zambon showed that the space of coisotropic deformations $\mathcal{C}$ 'near' a fixed coisotropic submanifold $C$ cannot carry the structure of a (Fréchet) manifold because there exist 'tangent vectors' whose sum is not tangent to $\mathcal{C}$. Oh and Park showed that this can be understood with the help of the $L_{\infty}$-algebra on the sections of the normal bundle to $C$ : the extension of elements in the first algebroid cohomology to Maurer-Cartan elements meets several obstructions, and the first of them is given by a quadratic relation. Hence, the sum of solutions might fail to be a solution again which explains M. Zambon's observation.

Consider the manifold $M=\mathbb{R}^{2} \times\left(S^{1}\right)^{4}$ with coordinates $\left(x^{1}, x^{2}, \theta^{1}, \theta^{2}, \theta^{3}, \theta^{4}\right)(\theta$ denotes the angle-coordinate on $S^{1}$ ). We equip $M$ with the symplectic form

$$
\omega=d \theta^{1} \wedge d x^{1}+d \theta^{2} \wedge d x^{2}+d \theta^{3} \wedge d \theta^{4}
$$

and define $C:=\left(S^{1}\right)^{4}$ which is a coisotropic submanifold of $M$. The BFV-complex for $C$ is given by introducing a pair of ghosts $\left(c_{1}, c_{2}\right)$ and ghost-momenta $\left(b^{1}, b^{2}\right)$ for the transversal coordinates $\left(x^{1}, x^{2}\right)$. The element $\Omega_{0}$ reads $c_{1} x^{1}+c_{2} x^{2}$ and 
it is closed with respect to the total Poisson bracket on the BFV-complex, so no extension is needed. The total differential of the BFV-complex is given by

$$
D=x^{1} \frac{\partial}{\partial b^{1}}+x^{2} \frac{\partial}{\partial b^{2}}+c^{1} \frac{\partial}{\partial \theta^{1}}+c^{2} \frac{\partial}{\partial \theta^{2}}
$$

with homotopy

$$
h=b^{1} \frac{\partial}{\partial x^{1}}+b^{2} \frac{\partial}{\partial x^{2}}+\theta^{1} \frac{\partial}{\partial c^{1}}+\theta^{2} \frac{\partial}{\partial c^{2}} .
$$

The homotopy $h$ is a differential itself and if follows that it has the same cohomology as $D$. Moreover, $h$ can be interpreted as the de Rham differential on $\mathbb{R}^{2} \times\left(S^{1}\right)^{2}$ and consequently the cohomology with respect to $D$ is given by periodic functions in the variables $\theta^{3}$ and $\theta^{4}$ tensored by the Grassmann-algebra generated by $c^{1}$ and $c^{2}$.

The equation for Maurer-Cartan elements reads

$$
\left\{\Omega_{0}+F, \Omega_{0}+F\right\}=0
$$

and if we assume that $F$ is an element of $D$-cohomology this equation reduces to

$$
\{F, F\}_{C}=0
$$

where

$$
\{f, g\}_{C}:=\frac{\partial f}{\partial \theta^{4}} \frac{\partial g}{\partial \theta^{3}}-\frac{\partial f}{\partial \theta^{3}} \frac{\partial g}{\partial \theta^{4}} .
$$

Condition (22) was also found in [OP].

Consider a (formal) geometric Maurer-Cartan element $c_{1} f^{1}+c_{2} f^{2}$ where $f^{1}$ and $f^{2}$ are functions that depend on the angle-coordinates $\left(\theta^{1}, \ldots, \theta^{4}\right)$ only. When does this section of $N C$ define a coisotropic submanifold? We showed that this is equivalent to

$$
\left\{\Omega_{0}+c_{1} f^{1}+c_{2} f^{2}, \Omega_{0}+c_{1} f^{1}+c_{2} f^{2}\right\}_{M}
$$

being exact with respect to $\delta_{f}:=\left(x^{1}+f^{1}\right) \frac{\partial}{\partial b^{1}}+\left(x^{2}+f^{2}\right) \frac{\partial}{\partial b^{2}}$. Computing the bracket (23) yields

$$
2 c_{1} c_{2}\left(\frac{\partial f^{1}}{\partial \theta^{2}}-\frac{\partial f^{2}}{\partial \theta^{1}}+\frac{\partial f^{1}}{\partial \theta^{4}} \frac{\partial f^{2}}{\partial \theta^{3}}-\frac{\partial f^{2}}{\partial \theta^{4}} \frac{\partial f^{1}}{\partial \theta^{3}}\right) .
$$

We denote this expression by $H$. It only depends on the angle-variables. Exactness of $H$ translates into the condition that there exists a pair of functions $g_{1}$ and $g_{2}$ that might depend on all variables on $M$ such that $\delta_{f}\left(b^{1} g_{1}+b^{2} g_{2}\right)=\left(x^{1}+f^{1}\right) g_{1}+$ $\left(x^{2}+f^{2}\right) g_{2}=H$. In lowest order of $\epsilon$ this implies

$$
x^{1} g_{1}^{1}+x^{2} g_{2}^{1}=H^{1}
$$

where the superscripts at $g_{1}, g_{2}$ and $H$ indicate that we are considering the parts that are of order $\epsilon$ only. It follows that both sides of (24) must vanish separately. Hence $\delta_{0}\left(b^{1} g_{1}^{1}+b^{2} g_{2}^{1}\right)=0$ and since the cohomology of $\delta_{0}$ is concentrated in ghostmomentum degree $0, b^{1} g_{1}^{1}+b^{2} g_{2}$ is not only $\delta_{0}$-closed but even $\delta_{0}$-exact, i.e. it can be expressed as $\delta_{0}(\mu)$ for some $\mu$. We add $-\delta_{f}(\mu)$ to $b^{1} g_{1}+b^{2} g_{2}$ and observe that the new element starts at order $\epsilon^{2}$. Inductively one proves that one can choose $g_{1}$ and $g_{2}$ to be vanishing identically. Hence a section of the normal bundle to $C$ given by $c_{1} f^{1}+c_{2} f^{2}$ defines a coisotropic submanifold iff

$$
\frac{\partial f^{1}}{\partial \theta^{2}}-\frac{\partial f^{2}}{\partial \theta^{1}}+\frac{\partial f^{1}}{\partial \theta^{4}} \frac{\partial f^{2}}{\partial \theta^{3}}-\frac{\partial f^{2}}{\partial \theta^{4}} \frac{\partial f^{1}}{\partial \theta^{3}}=0 .
$$


This condition coincides with the one given in [Z, where it was derived in a more analytical context. (There is a difference in signs which is caused by the fact that the perturbed constraints should by defined as $y^{\mu}-f^{\mu}$ and not $y^{\mu}+f^{\mu}$ from the geometric point of view.)

\section{Appendix A. Proof of Lemma 2}

Instead of proving Lemma 2 directly we develop some kind of extended graphical calculus in $\mathbb{L}_{d}$. First we add lines that are marked by exactly one $\circ$ or one $\times$. We decorate marked edges by two natural numbers $\geq 0$ - one is placed at the segment before the mark, one on the segment after it:

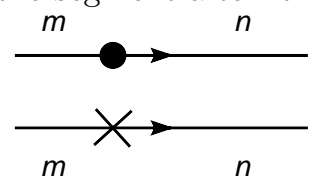

We extend $\Phi$ to diagrams of this kind. The only change we make is that instead of composing $\Phi$ (line decorated by $m$ ) and $\Phi$ (line decorated by $n$ ) via $\circ$ ho at the special edge we use $\circ \Pi_{H} \circ$ for the pointed edge and ordinary composition for the edge with a cross. We call diagrams of the first kind 'pointed' and diagrams of the second kind 'truncated'.

The crucial step is to realize that

$$
\delta_{0} \circ \widetilde{\mathcal{D}_{\epsilon}} \circ \iota=\widetilde{\mathcal{D}_{\epsilon}} \circ \mathcal{D}_{\epsilon}
$$

because then $\mathcal{D}_{\epsilon} \circ \mathcal{D}_{\epsilon}=\Pi_{H} \circ \delta_{0}(\cdots)=0$.

To prove this we have to compute $\delta_{0} \circ \Phi$ (line decorated by $n$ ) $\iota$. We use equation (77) and

$$
\left[\delta_{0}, D_{R}\right]=-D_{R} \circ D_{R}
$$

which is an immediate consequence of the fact that $D_{\epsilon}:=\delta_{0}+D_{R}$ is a coboundary operator. Consequently

$$
\begin{aligned}
& \delta_{0} \circ \Phi(\stackrel{n}{\longrightarrow})=\delta_{0} \circ-D_{R} \circ h \circ \Phi(\stackrel{n-1}{\longrightarrow})= \\
& =D_{R} \circ D_{R} \circ h \circ \Phi(\stackrel{n-1}{\longrightarrow})+D_{R} \circ \delta_{0} \circ h \circ \Phi(\stackrel{n-1}{\longrightarrow})= \\
& =D_{R} \circ D_{R} \circ h \circ \Phi(\stackrel{n-1}{\longrightarrow})+D_{R} \circ \Phi(\stackrel{n-1}{\longrightarrow})+ \\
& +\left(-D_{R} \circ \Pi_{H} \circ \Phi(\stackrel{n-1}{\rightarrow})-D_{R} \circ h \circ \delta_{0} \circ \Phi(\stackrel{n-1}{\longrightarrow})\right)= \\
& =\Phi(\stackrel{*}{*})-\Phi\left(\star^{n-1}\right)+ \\
& +\Phi(\stackrel{1}{\rightarrow} \stackrel{n-1}{\longrightarrow})+\Phi(\stackrel{1}{\rightarrow}) \circ h \circ \delta_{0} \circ \Phi(\stackrel{n-1}{\longrightarrow})
\end{aligned}
$$

Inductively it follows that

$$
\delta_{0} \circ \Phi(\stackrel{n}{\longrightarrow}) \circ \iota=\left(\sum_{r+s=n+1} \Phi(\stackrel{r}{\longleftarrow} s\right.
$$


since $\delta_{0}$ always moves on the right and produces new contributions that have interpretations in our graphical calculus until it hits $\iota$ where it vanishes. If we consider

$$
\sum_{n \geq 1} \delta_{0} \circ \Phi(\stackrel{n}{\longrightarrow}) \circ \iota
$$

all the terms containing truncated diagrams vanish and we are left with

$$
\delta_{0} \circ \widetilde{\mathcal{D}_{\epsilon}} \circ \iota=\sum_{r, s} \Phi(\stackrel{r}{\rightarrow} s
$$

and when we compose this identity from the left with $\Pi_{H}$ we obtain the first part of Lemma 2 ,

Next we prove that

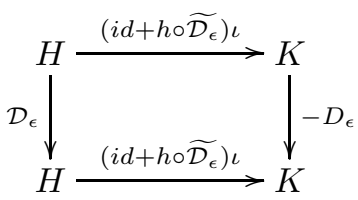

commutes. We will always assume that we restrict the arguments to $H$, so we can compute

$$
\begin{aligned}
-D_{\epsilon}\left(i d+h \circ \widetilde{\mathcal{D}_{\epsilon}}\right) & =-\left(\delta_{0}+D_{R}\right)\left(i d+h \circ \widetilde{\mathcal{D}_{\epsilon}}\right)=-\delta_{0} \circ h \circ \widetilde{\mathcal{D}_{\epsilon}}+\widetilde{\mathcal{D}_{\epsilon}}= \\
& =\Pi_{H} \circ \widetilde{\mathcal{D}_{\epsilon}}+h \circ \delta_{0} \circ \widetilde{\mathcal{D}_{\epsilon}}=\Pi_{H} \circ \widetilde{\mathcal{D}_{\epsilon}}+h \circ \widetilde{\mathcal{D}_{\epsilon}} \circ \mathcal{D}_{\epsilon} \\
& =\left(i d+h \circ \widetilde{\mathcal{D}_{\epsilon}}\right) \circ \mathcal{D}_{\epsilon} .
\end{aligned}
$$

Notice that we had to add the minus sign. If we want a chain map between $\left(H, \mathcal{D}_{\epsilon}\right)$ and $\left(K, D_{\epsilon}\right)$ we start our construction not from $D_{\epsilon}$ but from $-D_{\epsilon}$ instead. The chain map $\left(H, \mathcal{D}_{\epsilon}\right) \rightarrow\left(K,-D_{\epsilon}\right)$ induces an isomorphism in cohomology, because is a perturbation of a quasi-isomorphism, hence it is quasi-isomorphism itself. See GL for the details.

\section{Appendix B. Proof of Lemma 3}

We prove Lemma 3 inductively. The first sheet is $H\left(K, \delta_{0}\right)$ for both the spectral sequence and in our construction and the differentials are the same (up to the usual minus sign). So we can assume that the constructions yield the same spaces and differentials up to the $n$ 'th sheet. We have to prove that the two differentials defined on $E_{n}$ coincide (again up to sign). Take an element $[x]_{n} \in E_{n}$ (the subscript indicates that we consider $x$ as an element of the $n$ 'th sheet of the spectral sequence here). But this means that there are elements $y_{1}, \cdots, y_{n-1}$ such that

$$
\begin{aligned}
\delta_{0} x & =0, \\
\delta_{1} x & =-\delta_{0} y_{1}, \\
\delta_{1} y_{1} & =-\delta_{0} y_{2}, \\
\cdots & \\
\delta_{1} y_{n-2} & =-\delta_{0} y_{n-1} .
\end{aligned}
$$

One can arrange these elements without loss of generality such that all of them lie in $A$. We compute the differential we obtain from the quantum coboundary 
operator:

$$
\begin{aligned}
\left(\delta_{1} \circ h\right)^{n-1} \circ \delta_{1}(x) & =\left(\delta_{1} \circ h\right)^{n-1} \circ-\delta_{0}\left(y_{1}\right) \\
& =\left(\delta_{1} \circ h\right)^{n-2} \circ \delta_{1} \circ-\left(h \circ \delta_{0}\right)\left(y_{1}\right) \\
& =\left(\delta_{1} \circ h\right)^{n-1} \circ \delta_{1}\left(-y_{1}-\delta_{0} \circ h\left(y_{1}\right)\right)
\end{aligned}
$$

and it is easy to check that the second part in the last line disappears because it is exact with respect to the differential on the $E_{n-1}$-sheet. On all the next steps the same happens: we produce two terms, one contains the next $y$ from our sequences and the other one is exact with respect to one of the differentials from a previous sheet of the spectral sequence and so it does not contribute. In the last step we arrive at

$$
\delta_{1} y_{n-1}
$$

(up to sign). But this is exactly the differential in the $n$ 'th sheet of the spectral sequence.

\section{Appendix C. Proof of Proposition 1}

First we have to adapt the graphical calculus that was introduced in Appendix A to the new situation. We start with introducing pointed and truncated diagrams in our framework. As before, one is allowed to add an o or $\times$ at one of the interior edges and extend $\Phi$ to this diagrams by replacing $\circ h \circ$ by $\circ \Pi_{h} \circ$ or ordinary composition in case of the special edge being marked by a circle or cross. Furthermore one has to add a sign. For example $\Phi$ of

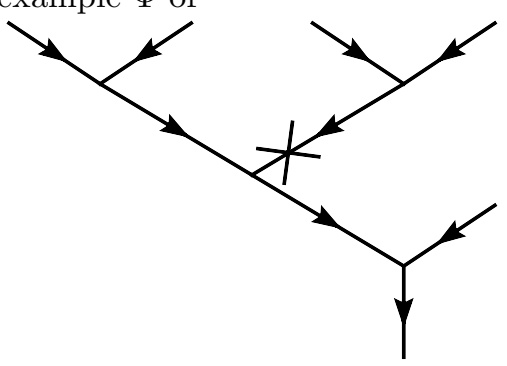

applied to $x_{1} \otimes \cdots x_{5}$ will get an additional sign given by $(-1)^{\left|x_{1}\right|+\left|x_{2}\right|}$. The general rule is to add the sign given by $(-1)$ to the powers of the sum of all inputs left to the truncated or pointed internal edge. We will denote the set of truncated diagrams by $\mathbb{T}^{\times}$and the pointed ones by $\mathbb{T}^{\circ}$.

There are four natural maps

$$
\begin{aligned}
F_{T}: \mathbb{T}^{\times} & \rightarrow \mathbb{T} \\
F_{P}: \mathbb{T}^{\circ} & \rightarrow \mathbb{T} \\
T: \mathbb{T} & \rightarrow \mathbb{T}^{\times} \\
P: \mathbb{T} & \rightarrow \mathbb{T}^{\circ}
\end{aligned}
$$

where $F_{T}$ maps a truncated tree to an untruncated one by forgetting that some edge is truncated and $F_{P}$ does the same for pointed diagrams. $T$ (the truncation map) maps an untruncated diagram to the sum of all possible truncations and $P$ does the same but by adding a point to the diagram. For example 


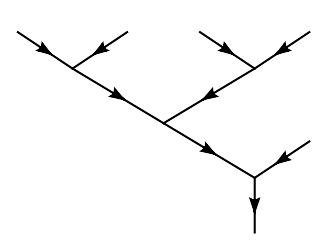

gets mapped to

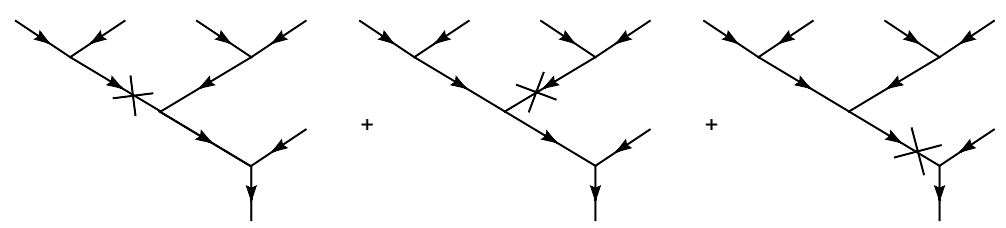

Furthermore there are two more maps that will be of importance later:

$$
\begin{aligned}
D(n): \mathbb{T}(n) & \rightarrow \bigoplus_{r+s=n} \mathbb{T}(r) \times \mathbb{T}(s) \\
C(r, s): \mathbb{T}(r) \times \mathbb{T}(s) & \rightarrow \mathbb{T}(r+s)
\end{aligned}
$$

where $D$ (decomposition map) simply decomposes the tree at the trivalent vertex near its root and $C$ joins two trees by adding one more trivalent vertex and sticking the two trees into the two incoming edges. One can extend $D$ to the case of truncated / pointed diagrams by simply cutting the truncated / pointed diagram at the marked edge.

The central claim is the following

Lemma 5. When restricted to arguments in $T(H)$ the following identity holds for every oriented tree $F \in \mathbb{T}$ :

$$
d(\Phi(F))=\Phi(P(F))-\Phi(T(F)) .
$$

Proof. This follows easily by induction over the number of leaves of the trees. We start with $\mathbb{T}(2)$ which only contains one element, namely

and we obtain

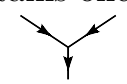

$$
d(\Phi(Y))\left(x_{1} \otimes x_{2}\right)=\quad d\left[x_{1}, x_{2}\right]_{\epsilon}=-\left[d x_{1}, x_{2}\right]_{\epsilon}-(-1)^{\left|x_{1}\right|}\left[x_{1}, d x_{2}\right]_{\epsilon}=0
$$

since $x_{1}$ and $x_{2}$ are in cohomology. But this is what we claimed above because there are not internal edges contained in this diagram.

To make the inductive step we start with a tree and apply the decomposition map $D$. One easily arrives at

$$
\begin{aligned}
& d\left(\Phi\left(x^{G}\right)\right)\left(x_{1} \otimes \cdots \otimes x_{s} \otimes x_{s+1} \otimes \cdots \otimes x_{n}\right)= \\
& +\left[h \circ d \circ \Phi(F)\left(x_{1} \otimes \cdots \otimes x_{s}\right), h \circ \Phi(G)\left(x_{s+1} \otimes \cdots \otimes x_{n}\right)\right] \\
& +(-1)^{\left(\left|x_{1}\right|+\cdots+\left|x_{s}\right|\right)}\left[h \circ \Phi(F)\left(x_{1} \otimes \cdots \otimes x_{s}\right), h \circ d \circ \Phi(G)\left(x_{s+1} \otimes \cdots \otimes x_{n}\right)\right]
\end{aligned}
$$


We can apply our induction hypothesis on the last two terms because $F$ and $G$ have certainly fewer inputs than the diagram we started with and one arrives at the claimed formula.

Now we can prove that we really get a new $L_{\infty}$-algebra structure on $H$ as stated in Proposition 1] We will use that one obtains the following explicit formula for the Jacobiators after some computations:

$$
\begin{aligned}
J(n)\left(x_{1} \otimes \cdots \otimes x_{n}\right)= & \sum_{\sigma \in \Sigma_{n}} \operatorname{sign}(\sigma) \sum_{[F] \in[\mathbb{T}(n)]} \frac{1}{|\operatorname{Aut}([F])|} . \\
& \Pi_{H} \Phi(P(F))\left(x_{\sigma(1)} \otimes \cdots \otimes x_{\sigma_{n}}\right) .
\end{aligned}
$$

By identity (25) from Lemma 5 it follows that we can replace $\Phi(P(F))$ by $\Phi(T(F))$ in the above formula since the $d(\cdots)$-part disappears when we apply $\Pi_{H}$. Next we demonstrate that

$$
\sum_{\sigma \in \Sigma_{n}} \operatorname{sign}(\sigma) \sum_{[F] \in[\mathbb{T}(n)]} \frac{1}{|\operatorname{Aut}([F])|} \Pi_{H} \Phi(T(F))=0
$$

and so our claim will follow.

The crucial part is to understand why the diagrams with a cross at one of the two lowest internal vertices do not contribute to the sum (26). So we consider the decomposition of $F$ into two parts and the corresponding truncated diagrams:

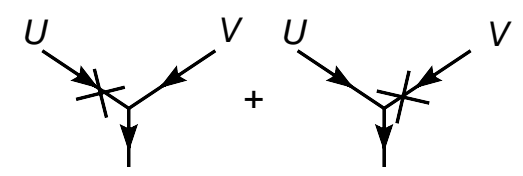

The contribution of diagrams like this is

$$
\begin{aligned}
& =\sum_{\sigma \in \Sigma_{n}} \operatorname{sign}(\sigma) \sum_{[F] \in[\mathbb{T}(n)]} \frac{1}{\mid \operatorname{Aut}([F])} \Pi_{H} \Phi\left(T\left({ }^{*}+x^{v}\right)\right)\left(x_{\sigma(1)} \otimes \cdots \otimes x_{\sigma(n)}\right) \\
& =\sum_{\sigma \in \Sigma_{n}} \operatorname{sign}(\sigma) \sum_{r+s=n} \sum_{[U] \in[\mathbb{T}(r)],[V] \in[\mathbb{T}(s)]} \frac{1}{|\operatorname{Aut}[U]||\operatorname{Aut}[V]|} \\
& \times\left(\left[\Phi(U)\left(x_{\sigma(1)} \otimes \cdots \otimes x_{\sigma(s)}\right), h \circ \Phi(V)\left(x_{\sigma(s+1)} \otimes \cdots \otimes x_{\sigma(n)}\right)\right]\right) \\
& =\sum_{\sigma \in \Sigma_{n}} \operatorname{sign}(\sigma) \sum_{r+s+t=n} 1 / 2 \sum_{[U] \in[\mathbb{T}(r)],[V] \in[\mathbb{T}(s)],[W] \in[\mathbb{T}(t)]} \frac{1}{|\operatorname{Aut}[U]||\operatorname{Aut}[V]||\operatorname{Aut}[W]|} \\
& \times\left(\left[\left[h \circ \Phi(U)\left(x_{\sigma(1)} \cdots x_{\sigma(r)}\right), h \circ \Phi(V)\left(x_{\sigma(r+1)} \cdots x_{\sigma(r+s)}\right)\right],\right.\right. \\
& \left.\left.h \circ \Phi(W)\left(x_{\sigma(r+s+1)} \cdots x_{\sigma(n)}\right)\right]\right)
\end{aligned}
$$

and this vanishes by Jacobi-identity since the last line is of the form $[[a, b], c]$ and we sum over all permutations with appropriate signs.

The same argument works for the other truncated diagrams: fix the diagram below the truncation point and put it together with the term where the truncation point lies on the opposite side. One can apply the argument from above by taking the partial sum where the diagram below the truncated edge stays fixed and then 
everything reduces to the Jacobi-identity again.

Next we prove the statement that one obtains an $L_{\infty}$-quasi-isomorphism between the constructed $L_{\infty}$-algebra on cohomology and $(K,-d, \epsilon[\cdot, \cdot])$. Since we start with an $L_{\infty}$-quasi-isomorphism for $\epsilon=0$ which is given by $\iota$, we have natural candidates for a perturbation of this quasi-isomorphism

$$
\psi_{n}:=h \circ \widetilde{M_{n}} \circ \iota: S^{n}(H) \rightarrow K
$$

that should be an $L_{\infty}$-morphism. Since $\widetilde{M}_{1}=0$ and because there is no induced differential on $H$ the first component $\iota+\psi_{1}$ is just $\iota: H \rightarrow K$ which is still a chain map that induces an isomorphism on cohomology. So when we can prove that this perturbation of $\iota$ is an $L_{\infty}$-morphism it automatically will be an $L_{\infty}$ quasi-isomorphism.

We give explicit formulas for the identities that must be checked in order to prove that we obtain an $L_{\infty}$-morphism (we suppress $\iota$ ): $I(n): S^{n}(H) \rightarrow K$ given by

$$
\begin{aligned}
& -d\left(h \circ \widetilde{M_{n}}\left(x_{1} \otimes \cdots \otimes x_{n}\right)\right) \\
& +1 / 2 \sum_{r+s=n} \sum_{\sigma \in(r, s)-\text { shuffles }} \operatorname{sign}(\sigma)\left[h \circ \widetilde{M}_{r}\left(x_{\sigma(1)} \otimes \cdots \otimes x_{\sigma(r)}\right),\right. \\
& -\sum_{p+q=n} \sum_{\tau \in(q, p)-\text { shuffles }} \operatorname{sign}(\sigma) h \circ \widetilde{M_{p+1}}\left(\Pi_{H} \circ \widetilde{M}_{q}\left(x_{\tau(1)} \otimes \cdots \otimes x_{\tau(q)}\right) \otimes\right. \\
& \left.\otimes x_{\tau(q+1)} \otimes \cdots \otimes x_{\tau(n)}\right)
\end{aligned}
$$

has to vanish identically for all $n \geq 2$. One can easily prove that the middle term is equal to

$$
\widetilde{M_{n}}\left(x_{1} \otimes \cdots \otimes x_{n}\right)
$$

and inserting the defining formula for the homotopy into the term in the first line gives

$$
\left.-\widetilde{M_{n}}\left(x_{1} \otimes \cdots \otimes x_{n}\right)\right)+\Pi_{H} \widetilde{M_{n}}\left(x_{1} \otimes \cdots \otimes x_{n}\right)+h \circ d\left(\widetilde{M_{n}}\left(x_{1} \otimes \cdots \otimes x_{n}\right)\right) .
$$

The first term of this expression cancels with $\tilde{M}_{n}\left(x_{1} \otimes \cdots \otimes x_{n}\right)$ that we obtained from the second line of $I(n)$. It remains to prove that the remaining three terms

$$
\begin{aligned}
& +\Pi_{H} \widetilde{M_{n}}\left(x_{1} \otimes \cdots \otimes x_{n}\right) \\
& +h \circ d\left(\widetilde{M_{n}}\left(x_{1} \otimes \cdots \otimes x_{n}\right)\right) \\
& -\sum_{p+q=n} \sum_{\tau \in(q, p)-\text { shuffles }} \operatorname{sign}(\sigma) h \circ \widetilde{M_{p+1}}\left(\Pi_{H} \circ \widetilde{M}_{q}\left(x_{\tau(1)} \otimes \cdots \otimes x_{\tau(q)}\right) \otimes\right. \\
& \left.\otimes x_{\tau(q+1)} \otimes \cdots \otimes x_{\tau(n)}\right)
\end{aligned}
$$

sum to zero. But this is not hard: we know from Lemma 5 that $d(($ tree $))$ is given by the sum over all pointed diagrams minus truncated diagrams. We proved before that the weighted sum over all unoriented diagrams and over permutations 
of the truncated diagrams vanishes. So we are left with the weighted sum over all pointed diagrams. However, the third term above is exactly the sum over all such contributions from pointed diagrams (plus a term from $\iota 0$ that we suppressed but that expression cancels with $\left.\Pi_{H} \widetilde{M_{n}}\left(x_{1} \otimes \cdots \otimes x_{n}\right)\right)$ so everything cancels and we are done.

\section{Appendix D. Proof of Theorem 2}

First we augment the set of trees by adding pointed and truncated ones. As before (see Appendix A and C) the 'composition' oho at the pointed / truncated interior edge is replaced by $\circ \Pi_{H} \circ /$ ordinary composition and one adds the sign given by $(-1)$ to the power of the sum over all entries that are left to the marked edge.

Lemma 6. Fix an oriented undecorated tree $F \in \mathbb{T}$. Then

$$
\sum_{\text {length }} \delta_{0} \Phi(F)=\sum_{\text {length }} \Phi(P(F))-\sum_{G \in \mathcal{A}(F)} \Phi(G)
$$

where we sum over all possible decorations of the undecorated tree $F$ in the first two summands and $\mathcal{A}$ denotes all decorations of $F$ with one truncated internal edge that is decorated by $(0,0)$.

Proof. First consider

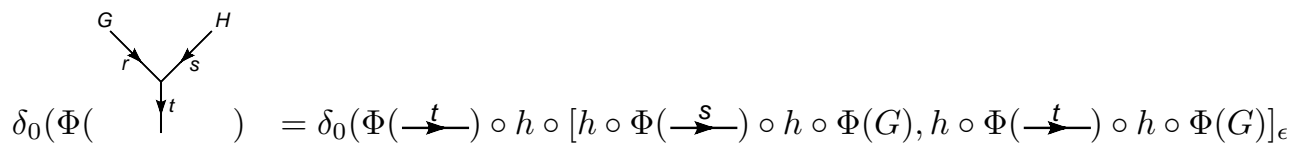

The calculation of $\delta_{0}(\Phi(\stackrel{r}{\rightarrow}))$ was performed in the Proof of Lemma 2 where it was the sum over contributions of all truncated lines of total length $r+1$ minus the sum over contributions of truncated diagrams of total length $r$. Moreover we compute

$$
\begin{aligned}
\delta_{0}[a, b]_{\epsilon} & =\left(\delta_{0}+D_{R}\right)[a, b]_{\epsilon}-D_{R}[a, b]_{\epsilon} \\
& =-\left[D_{R} a, b\right]_{\epsilon}-(-1)^{|a|}\left[a, D_{b}\right]_{\epsilon}-D_{R}[a, b]_{\epsilon}-\left[\delta_{0} a, b\right]_{\epsilon}-(-1)^{|a|}\left[a, \delta_{0} b\right]_{\epsilon}
\end{aligned}
$$

and when one combines these two results (and uses $\delta_{0} \circ h+h \circ \delta_{0}=1-\Pi_{H}$ successively) one obtains all pointed diagrams and observes that the contribution of any diagram containing a truncated edges on a decorated line decorated by something different from $(0,0)$ cancels with the contribution of some other decorated diagram when one sums over all decorations. So one is left with the claimed identity.

Now we can prove Theorem 2 ;

Proof. Similar to the proof of Proposition 1 we use the fact that the Jacobiators can be written as

$$
\begin{array}{r}
J(n)\left(x_{1} \otimes \cdots \otimes x_{n}\right)=\sum_{\sigma \in \Sigma_{n}} \operatorname{sign}(\sigma) \sum_{[F] \in\left[\mathbb{T}_{d}(n)\right]} \frac{1}{|\operatorname{Aut}([F])|} . \\
\Pi_{H} \Phi(P(F))\left(x_{\sigma(1)} \otimes \cdots \otimes x_{\sigma_{n}}\right)
\end{array}
$$


We apply Lemma 6 and obtain that the Jacobiators are equal to

$$
\sum_{\sigma \in \Sigma_{n}} \operatorname{sign}(\sigma) \sum_{G \in \mathcal{A}(F): F \in\left[\mathbb{T}_{d}(n)\right]} \frac{1}{|\operatorname{Aut}([F])|} \Pi_{H} \Phi(G)\left(x_{\sigma(1)} \otimes \cdots \otimes x_{\sigma_{n}}\right) .
$$

But this sum vanishes because of the Jacobi identity: fix the diagram 'below' the truncation point and consider the corresponding diagram with the truncation point on the other side of the branch. Taking their contributions together one can write it as a sum over contributions coming from unoriented diagrams attached to the trivalent vertex with one truncated incoming edge and an ordinary incoming edge. Since the truncated edge is decorated by zeros the diagram attached to the truncated incoming edge can also be written as $(1 / 2$ of $)$ the sum of two more unoriented diagrams attached to a trivalent vertex. It follows from Jacobi identity that the sum over all three diagrams and over all permutations vanishes. Basically this is the same argument we used to prove Proposition 1

The proof that this induced structure is $L_{\infty}$-quasi-isomorphic to $\left(K,-D_{\epsilon},[\cdot, \cdot]_{\epsilon}\right)$ can be copied verbatim from the proof we gave for Proposition 1] again we obtain a sum that contains the sum over all truncated and all pointed diagrams minus the sum over all pointed diagrams. Since the sum over all truncated diagrams vanishes as demonstrated above, we are done.

\section{Appendix E. Globalisation of Theorem 4}

In the following we explain a globalisation of the BFV-complex essentially due to M. Bordemann and H.-C. Herbig (see $[\mathrm{B}]$ and $[\mathrm{H}]$ ) and demonstrate that Theorem 4 extends to this setting.

As before one fixes an embedding of $N C$ into $M$ by which $N C$ inherits a Poisson bracket. We denote the projection of $N C$ to $C$ by $p$. Next one pulls back the bundle $N^{*}[1] C \oplus N[-1] C$ to $N C$ using $p$. This bundle carries a fibre metric given by the natural pairing between the fibres of $N C$ and $N^{*} C$, which we denote by $\{\cdot, \cdot\}_{g}$ again. One chooses a connection on $N C$ as a bundle over $C$, hence $N^{*} C$ also obtains a connection as does the Whitney sum of these two bundles. One pulls back this connection to $p^{*}\left(N^{*}[1] C \oplus N[-1] C\right)$. Next one extends the Poisson bivector field $\Pi$ on $N C$ to the total space of the bundle $p^{*}\left(N^{*}[1] C \oplus N[-1] C\right)$ with help of the connection. The sum of the bivector field $G$ given by $\{\cdot, \cdot\}_{g}$ and the bivector field one obtained by extending $\Pi$ using the connection does not form a Poisson bivector field. However, one can show that there exists a natural extension $\tilde{\Pi}$ of this sum that is Poisson. Rothstein gave an explicit expression for such an extension in local coordinates (see [Rot]) in the symplectic setting and $[\mathrm{H}$ ] contains the calculation that the formula also works in the general case. We give a systematic proof of the existence of the extented bracket using homological perturbation theory: one considers the complex of vector fields on $p^{*}\left(N^{*}[1] C \oplus N[-1] C\right)$ with differential $[G, \cdot]$, where $[\cdot, \cdot]$ denotes the Schouten-Nijenhuis bracket. That the extension $\tilde{\Pi}$ exists follows from the fact that the connection on $p^{*}\left(N^{*}[1] C \oplus N[-1] C\right)$ is metric with respect to $\{\cdot, \cdot\}_{g}$, that $\Pi$ is Poisson and that the cohomology of $[G, \cdot]$ is given by the vector fields on $N C$. Moreover one can prove that this extension is such that its part with ghost-momentum degree 1 is exactly $\{\cdot, \cdot\}_{g}$ and that its ghost-momentum 
degree 0 part evaluated on $\mathcal{C}^{\infty}\left(N^{*}[1] C\right)$ is given by

$$
\frac{1}{2} \Pi^{i j}\left(\frac{\vec{\partial}}{\partial x^{i}}+\Gamma_{i r}^{s} c_{s} \frac{\vec{\partial}}{\partial c_{r}}\right)\left(\frac{\vec{\partial}}{\partial x^{j}}+\Gamma_{j m}^{n} c_{n} \frac{\vec{\partial}}{\partial c_{m}}\right)
$$

where $x^{i}$ are coordiantes along $C$, the $c$ s denote linear coordinates along the fibres of $p^{*}\left(N^{*}[1] C\right)$, the expressions $\Pi^{i j}$ denote components of the Poisson bivector field $\Pi$ and the expressions $\Gamma_{i r}^{s}$ denote components of the Christoffel symbols associated to the chosen connection. We denote this part of the extended Poisson bivector field by $\{\cdot, \cdot\}_{E}$ and the total bracket corresponding to $\tilde{\Pi}$ by $\{\cdot, \cdot\}$. It has been observed by Herbig that the original argument of Stasheff $\mathrm{St}$ for the construction of $\Omega$ with $\{\Omega, \Omega\}=0$ does also work in the global setting, see $[\mathrm{H}]$. One decomposes the differential $\{\Omega, \cdot\}$ with respect to ghost degrees. The zero degree term is locally given by $\delta_{0}$ introduced in Subsection 3.1 and also the homotopy (13) is unchanged. One can compute $\delta_{1}$ restricted to $\mathcal{C}^{\infty}\left(N^{*}[1] C\right)$ which results into a global version of formula (15).

Next we want to consider the structure induced from the differential graded Poisson algebra $\left(\mathcal{C}^{\infty}\left(p^{*}\left(N^{*}[1] C \oplus N[-1] C\right)\right),\{\cdot, \cdot\},\{\Omega, \cdot\}\right)$ on the cohomology with respect to $\delta_{0}$. By the same reasoning as before with respect to the ghost-momentum degree one obtains that only the diagrams $\mathcal{R}(n)$ and $\mathcal{S}(n)$ contribute to the induced $L_{\infty}$-algebra structure on $\mathcal{C}^{\infty}\left(N^{*}[1] C\right)$ (see 21). However, $\{\cdot, \cdot\}_{M}$ gets replaced by $\{\cdot, \cdot\}_{E}$ in the definition of the map $R(n)$ associated to $\mathcal{R}(n)$ and $\delta_{1}$ gets replaced by its global version in the definition of the map $S(n)$ associated to $\mathcal{S}(n)$, see (16) and (17) and the explicit expressions for $R(n)$ and $S(n)$ given there.

We claim that the induced structure is exactly the $P_{\infty}$-algebra structure introduced by Oh and Park. To check this it suffices to compare the induced structure and the expressions for Oh and Park's structure in local charts. When we wrote down the expressions for the $P_{\infty}$-algebra structure on $\Gamma(\bigwedge N C)$ we implicitly assumed that we work with a connection that is trivial on the coordinate neighbourhood under consideration. But now we have already fixed a connection and use it to identify $T^{*}[1] N^{*}[1] C$ with $N^{*}[1] C \oplus N C \oplus T^{*}[1] C$. The formulas for the $P_{\infty}$-structure on $\mathcal{C}^{\infty}\left(N^{*}[1] C\right)$ read

$$
\begin{gathered}
\tau_{n}\left(c_{\nu_{1}} \otimes \cdots \otimes c_{\nu_{(n-2)}} \otimes f \otimes g\right)=\left.(-1)^{n}\left(\frac{\partial^{(n-2)}}{\partial y^{\nu_{1}} \cdots y^{\nu_{(n-2)}}}\{f, g\}\right)\right|_{C} \\
\tau_{n}\left(c_{\nu_{1}} \otimes \cdots \otimes c_{\nu_{(n-1)}} \otimes f\right)=(-1)^{n-1}\left(\frac { \partial ^ { ( n - 1 ) } } { \partial y ^ { \nu _ { 1 } } \cdots y ^ { \nu _ { ( n - 1 ) } } } \left(c_{\mu} \Pi^{\mu i} \frac{\partial f}{\partial x^{i}}+\right.\right. \\
\left.\left.+2 y^{i} \Gamma_{\alpha i}^{s} c_{s} \Pi^{\alpha r} \frac{\partial f}{\partial x^{r}}\right)\right)\left.\right|_{C} \\
+\left.(-1)^{n} \sum_{k=1}^{n-1}\left(\frac{\partial}{\partial y^{\nu_{1}}} \cdots \frac{\hat{\partial}}{\partial y^{\nu_{k}}} \cdots \frac{\partial}{\partial y^{(n-1)}}\left(\Pi^{i j} \Gamma_{i \nu_{k}}^{\beta} c_{\beta} \frac{\partial f}{\partial x^{i}}\right)\right)\right|_{C}
\end{gathered}
$$




$$
\begin{aligned}
\tau_{n}\left(c_{\nu_{1}} \otimes \cdots \otimes c_{\nu_{n}}\right)=(-1)^{n}\left(\frac { \partial ^ { n } } { \partial y ^ { \nu _ { 1 } } \cdots y ^ { \nu _ { n } } } \left(\frac{1}{2} \Pi^{\mu \lambda} c_{\mu} c_{\lambda}+\Gamma_{i \alpha}^{\beta} c_{\beta} y^{\alpha} \Pi^{i \mu} c_{\mu}+\right.\right. \\
\left.\left.+\frac{1}{2} \Gamma_{i \alpha}^{\beta} c_{\beta} y^{\alpha} \Pi^{i j} \Gamma_{j m}^{n} c_{n} y^{m}\right)\right)\left.\right|_{C} \\
+\left.(-1)^{(n-1)} \sum_{k=1}^{n}\left(\frac{\partial}{\partial y^{\nu_{1}}} \cdots \frac{\hat{\partial}}{\partial y^{\nu_{k}}} \cdots \frac{\partial}{\partial y^{\nu_{n}}}\left(\Pi^{i j} \Gamma_{i \nu_{k}}^{\beta} c_{\beta} \Gamma_{j m}^{n} y^{m} c_{n}+\Pi^{i \mu} \Gamma_{i \nu_{k}}^{\beta} c_{\beta} c_{\mu}\right)\right)\right|_{C} \\
+\left.(-1)^{n} \sum_{k<l}\left(\frac{\partial}{\partial y^{\nu_{1}}} \cdots \frac{\hat{\partial}}{\partial y^{\nu_{k}}} \cdots \frac{\hat{\partial}}{\partial y^{\nu_{l}}} \cdots \frac{\partial}{\partial y^{\nu_{n}}}\left(\Pi^{i j} \Gamma_{i \nu_{k}}^{\alpha} c_{\alpha} \Gamma_{j \nu_{l}}^{\beta} c_{\beta}\right)\right)\right|_{C}
\end{aligned}
$$

where $\wedge$ indicates that we leave out the corresponding partial derivatives, $y^{\mu}$ are coordinates of the tubular neigbourhood of $C$ transversal to $C$ and dual to $c_{\mu}$ and $f$ and $g$ are arbitrary functions on $C$.

One checks by direct computation similar to the one explained in Subsection 3.2 that these formulas coincide with the ones defining the induced structure. Moreover one observes that the structure maps of the induced $L_{\infty}$-algebra are multiderivations as before. By the same arguments as given in Subsection 3.2 for the local case it follows that the induced $L_{\infty}$-algebra is again exactly the one defined by $\mathrm{Oh}$ and Park, thus proving Theorem 4 in full generality.

\section{REFERENCES}

[BF] I.A. Batalin, E.S. Fradkin, A generalized canonical formalism and quantization of reducible gauge theories, Phys. Lett. 122B (1983), 157-164

[BV] I.A. Batalin, G.S. Vilkovisky, Relativistic S-matrix of dynamical systems with bosons and fermion constraints, Phys. Lett. 69B (1977) 309-312

[BB] R.E. Borcherds, A. Barnard, Lectures on Quantum Field Theory, lecture notes, math-ph/0204014

[B] M. Bordemann, The deformation quantization of certain super-Poisson brackets and BRST cohomology, math.QA/0003218

[BT] R. Bott, L. Tu, Differential Forms in algebraic Topology, Graduate Texts in Mathematics 82, Springer (1982)

[C1] A.S. Cattaneo, On the BV formalism, unpublished notes, http://www .math.unizh.ch/reports/07_05.pdf

[C2] A.S. Cattaneo, From Topological Field Theory to Deformation Quantization and Reduction, to appear in the proceedings of ICM 2006, http://math.unizh.ch/fileadmin/math/preprints/06-06.pdf

[CF] A.S. Cattaneo, G. Felder, Relative formality theorem and quantisation of coisotropic submanifolds, to appear in Adv. Math., math.QA/0501540

[CFL] A.S. Cattaneo, D. Fiorenza, R. Longoni, Graded Poisson Algebras, in Encyclopedia of Mathematical Physics, eds. J.-P. Françoise, G.L. Naber, S.T. Tsou, vol. 2, p. 560-567 (Oxford: Elsevier, 2006)

[G] E. Getzler, A Darboux theorem for Hamiltonian operators in the formal calculus of variations, Duke J. Math. 111 (2002), 535-560

[GL] A.K.A.M. Gugenheim, L.A. Lambe, Perturbation theory in differential homological algebra I, Il. J. Math., 33 (1989)

$[\mathrm{H}] \quad$ H.-C. Herbig, Variations on homological Reduction, PhD-Thesis at the University of Frankfurt, submitted 2006

[K] H. Khudaverdian, Semidensities on Odd Symplectic Supermanifolds, math.DG/0012256

[KS] M. Kontsevich, Y. Soibelman, Homological mirror Symmetry and torus fibrations, math.SG/0011041

[LSt] T. Lada, J. Stasheff, Introduction to sh Lie algebras for physicists, Int. J. Theor. Phys. 32 (1993), 1087-1104 
[L] A. Losev, BV formalism and quantum homtopical structures, series of three talks given at GAP 3 in Perugia (2006)

[LS] S.L. Lyakhovich, A.A. Sharapov, BRST theory without Hamiltonian and Lagrangian, J. High Energy Phys. JHEP03(2005)011, (2005)

[MSS] M. Markl, S. Shnider, J. Stasheff, Operads in Algebra, Topoolgy and Physics, volume 96 of Mathematical Surveys and Monographs. American Mathematical Society, Providence, Rhode Island (2002)

[Me] S.A. Merkulov, Strongly homotopy algebras of a Kähler manifold, Internat. Math. Res. Notices (1999), no.3, 153-164

[M] P. Mnëv, Notes on simplicial BF theory, hep-th/0610326

[OP] Y.G. Oh, J.S. Park, Deformations of coisotropic submanifolds and strong homotopy Lie algebroids, Invent. Math. 161 (2005), 287-36

[P] M. Polyak, Feynman diagrams for pedesterians and mathematicians, lecture notes for DennisFest, math.GT/0406251

[Rot] M. Rothstein, The structure of supersymplectic supermanifolds., in Differential Geometric Methods in Mathematical Physics, eds. C. Barecci et al., Proc. 19th Int. Conf., Rapallo/Italy 1990, Lect. Notes Phys. 375, 331-343 (1991)

[Roy] D. Roytenberg, Courant algebroids, derived brackets and even symplectic supermanifolds, PhD thesis, UC Berkeley (1999), math.DG/9910078

[Sw] A. S. Schwarz, Geometry of Batalin-Vilkovisky quantization, Commun. Math. Phys. 155 (1993), 249-260

[Se] P. Ševera, On the origin of the $B V$ operator on odd symplectic supermanifolds, math.DG/0506331

[St] J. Stasheff, Homological reduction of constrained Poisson algebras, J. Diff. Geom. 45 (1997), 221-240

[V] Th. Voronov, Higher derived brackets and homotopy algebras, math.QA/0304038

[Z] M. Zambon, Averaging techniques in Riemannian, symplectic and contact geometry, Ph.D. Thesis (University of Berkeley)

Institut für Mathematik, Universität ZÜrich-Irchel, Winterthurerstrasse 190, CH8057 ZÜRICH, SWITZERLAND

E-mail address: florian.schaetz@math.unizh.ch 